\title{
Hygro-thermo-mechanical bending of FG piezoelectric plates using quasi-3D shear and normal deformations theory
}

\author{
Ashraf M. Zenkour ${ }^{a, b^{*}}$ (iD \\ Zahra S. Hafed ${ }^{a, c}$ (iD) \\ a Department of Mathematics, Faculty of Science, King Abdulaziz University, Jeddah 21589, Saudi Arabia. E-mails: zenkour@kau.edu.sa, \\ zenkour@sci.kfs.edu.eg, zahra9879@yahoo.com \\ ${ }^{b}$ Department of Mathematics, Faculty of Science, Kafrelsheikh University, Kafrelsheikh 33516, Egypt \\ c Department of Mathematics, Faculty of Science, King Khaled University, Abha 21589, Saudi Arabia
}

*Corresponding author

http://dx.doi.org/10.1590/1679-78255396

\begin{abstract}
A simple quasi-3D sinusoidal shear and normal deformations theory for the hygro-thermo-mechanical bending of functionally graded piezoelectric (FGP) plate is developed under simply-supported edge conditions. The governing equations are deduced based on the principle of virtual work. The exact solutions for FGP plate are obtained. The current study investigates the effect of some parameters, like piezoelectricity, hygrothermal parameter, gradient index and electric loading on the mechanical and electric displacements, electric potential and stresses. They are explored analytically and numerically presented and discussed in detail. The numerical results clearly show the effect of piezoelectric and hygrothermal parameter on the FGP plate.
\end{abstract}

\section{Keywords}

Functionally graded, hygrothermal piezoelectric plates, bending, quasi-3D, analytical solutions.

\section{INTRODUCTION}

Piezoelectric materials are great widely used as smart structures in different aerospace applications, because they can generate voltage, drive microelectronics directly and store charge. The effect of piezoelectricity is clear through the linear electromechanical interaction between electrical and mechanical states. Piezoelectric materials are used to detect stresses and deformations. Also, these materials can exchange output electrical potential and input mechanical energy.

Zenkour (2014a, b) proposed an analytical solution describing the hygro-thermo-elastic responses of piezoelectric inhomogeneous hollow cylinders where the significance of influence of several parameters was investigated. He assumed that the piezoelectric nanoplate is simply-supported under an external electric voltage as well as a biaxial force and a uniform temperature change. The thermo-electro-mechanical free vibration of piezoelectric nanoplates based on the classical theory and nonlocal theory has been investigated by Liu et al. (2013). On the other hand, the thermo-electromechanical vibration of the piezoelectric rectangular nanoplate under different boundary conditions formed by using the nonlocal theory and the first-order shear deformation theory has been studied by Ke et al. (2015). Moreover, in the last decade, the analysis of static and dynamics for plates and shells made of piezoelectric materials have led to increase research attempts. In fact, the known theory for studying the mechanical behavior of rectangular plates is Kirchhoff classical plate theory (CPT). Jandaghian et al. $(2013,2014)$ presented exact solutions for the transient bending of a circular rectangular plate surface bounded by two piezoelectric layers by using CPT. Furthermore, Huang and Yu (2006) discussed the piezoelectricity model under the effect of piezoelectric surface layers. Consequently, a piezoelectric material under 
potential has been studied. As for the sinusoidal shear deformation plate model, it is presented by Touratier (1991) in the trigonometric higher-order shear deformation theory. The new theory is based on the kinematical way in which the shear is formed by a certain sine function.

Functionally graded plates (FG) are at present developed for common use as structural elements in extremely hightemperature gradient environments. Several researches have been conducted to analyze the mechanical or the thermal behavior of FG shells, beam and plates (Reddy and Chin 1998; Loy et al. 1999; Noda 1999; Cheng and Batra 2000a, b; Reddy 2000; Vel and Batra 2002; Qian and Batra 2004; Altenbach and Eremeyev 2008a, b; Lü et al. 2009; Ying et al. 2009; Bîrsan et al. 2012). Ootao and Tanigawa (2000) discussed the theoretical analysis of a three-dimensional transient piezo-thermo-elasticity for an FG plate with assuming this plate has nonhomogeneous mechanical and thermal material properties in the thickness direction. Lee and Saravanos (1997) verified a smart thermo-piezoelectric plate structures mechanics by using a generalized discrete layer theory. Afterwards, Cho and Oh (2004) developed a zig-zag higher-order plate smart theory for the mechanical, electric and thermal behaviors that are fully coupled. Tahani and Mirzababaee (2009) used layer-wise theories to analyze methods displacements and stresses of FG plates in cylindrical bending under thermo-mechanical loadings. In addition, a literature review of novel research works on FG plates was presented by Jha et al. (2013).

Since effects of transverse shear deformation are more cleared in thick plates or plates made of advanced composite materials as FGs, shear deformation theories that calculate the effects of shear deformation are often used to predict the responses of FG plates. The first-order shear deformation theory (FSDT) accounts for shear deformation effects, but this theory assumption violates the equilibrium conditions at the top and bottom surfaces of the plate. This shear deformation theory has been discussed by Mindlin (1957) and Reissner (1945). The higher-order shear deformation theories (HSDT) of laminated plates discussed by Reddy $(1984,2000)$ account for the in-plane displacements between layers through the thickness of the plate and satisfy the boundary conditions and equilibrium equations. The bending analysis and free vibration properties of FG rectangular plates may be treated in the literature by using a simple twovariable shear deformation theory that was investigated and presented by (Mechab et al. 2010; Zenkour and Sobhy 2015; Bellifa et al. 2016). Based on this theory, Zhang and Zhou (2008) studied free vibration, buckling and deflection of the FG thin plates. The dynamic response of initially stressed FG plates under transverse partially impulsive lateral distributed loads, without or resting on an elastic foundation, has been studied by Yang and Shen (2001). A new FSDT has been developed by Bellifa et al. (2016) to analyze bending and dynamic behaviors of FG rectangular plates.

The thermo-mechanical bending analysis of simply-supported FG plates resting on Winkler-Pasternak elastic foundations has been discussed by Bouderba et al. (2013). A new refined trigonometric shear deformation theory (RTSDT) has been developed. The trigonometric distribution of transverse shear deformation stress theory satisfies the free transverse shear stress distribution through the top and bottom surfaces for FG plate without using the shear deformation correction factors. This trigonometric higher-order deformation theory is developed to analyze bending and dynamic behaviors of FG rectangular plates. The equations governing the axial stress and transverse shear deformations of FG rectangular plates are studied by Bousahla et al. (2014). The bending response of FG plate that depend on elastic foundation and subjected to hygro-thermo-mechanical effects has been presented by Zidi et al. (2014). Lately, a refined trigonometric shear deformation theory (RTSDT) taking in consideration the effects of transverse shear deformation for the thermo-elastic bending analysis of FG sandwich plates was proposed by Tounsi et al. (2013). Zenkour (2006) studied the bending of an FG material plate for a simply-supported based on the sinusoidal shear deformation theory under a transverse uniform load.

Piezoelectric and elastic structures have been developed. These structures are commonly applied in changing thermal environment, most problems occur due to the changes in the material characteristic at the interfaces between layers. Thus, the structures made of functionally graded piezoelectric material (FGP) are developed. The transient bending analysis of circular FG plates incorporated with two uniformly sinusoidal distributed actuator layers made of piezoelectric material by using the CPT is discussed by Jafari et al. (2014). Li et al. (2014) developed the bending analysis and free vibration of FG piezoelectric beam model based on modified strain gradient and Timoshenko beam theories. The governing equations of new FGP beam model have been solved for a simply-supported beam. Beni (2016) studied the nonlinear formulation electromechanical bending, buckling, and free vibration analysis of FGP nanobeams by using the consistent size-dependent theory. The nonlinear governing equations of the present FGP nanobeam are derived by using Hamilton's principle and applying Euler-Bernoulli model.

In this article, the present quasi-3D shear and normal deformations plate theory is used for hygro-thermomechanical bending of FG piezoelectric plates. The effects of temperature field, moisture expansion, aspect ratio, electric loading, side-to-thickness ratio and volume fraction distribution on the deflection, electric potential, normal and transverse shear stresses of the FG piezoelectric plate are studied. Numerical analysis is presented to explain the validity 
and efficiency of the theory by comparing the obtained results with those computed using several other theories available in the literature.

\section{FORMULATION OF THE PROBLEM}

\subsection{Properties of FGP plate}

This article presents a simply-supported rectangular plate of length $a$, width $b$ and uniform thickness $h$ made of FGP material. The FGP plate is subjected to applied voltage $V_{e}$ at upper and lower surfaces, elevated temperature $T(x, y, z)$ as well as a moisture exposure $C(x, y, z)$ and sinusoidally distributed transverse load $q(x, y)$, as shown in Fig. 1 . Material properties of FGP plates are assumed to vary through thickness according to a power law distribution. Effective material properties $P(z)$ such as Young's modulus, moisture expansions and thermal expansions are supposed to vary continuously in the depth direction according to a power-law. Suppose that the FGP plate is made by mixing two different material phases, for instance, ceramic and metal, and are expressed as

$P(z)=P_{m}+\left(P_{c}-P_{m}\right)\left(\frac{z}{h}+\frac{1}{2}\right)^{k}$

where $P_{m}$ and $P_{c}$ are the properties of the metal and ceramic, respectively, and $k$ denotes the non-negative gradient index.

The material properties of the exponentially graded piezoelectric (EGP) plates are supposed to follow the exponential law and is expressed as (Zenkour 2007):

$P(z)=P_{0} \mathrm{e}^{k\left(\frac{z}{h}+\frac{1}{2}\right)}, k=\ln \left(\frac{P_{c}}{P_{m}}\right)$,

where $P_{m}=P_{0}$ is metal property and $P_{c}=P_{0} \mathrm{e}^{k}$ is the ceramic one.
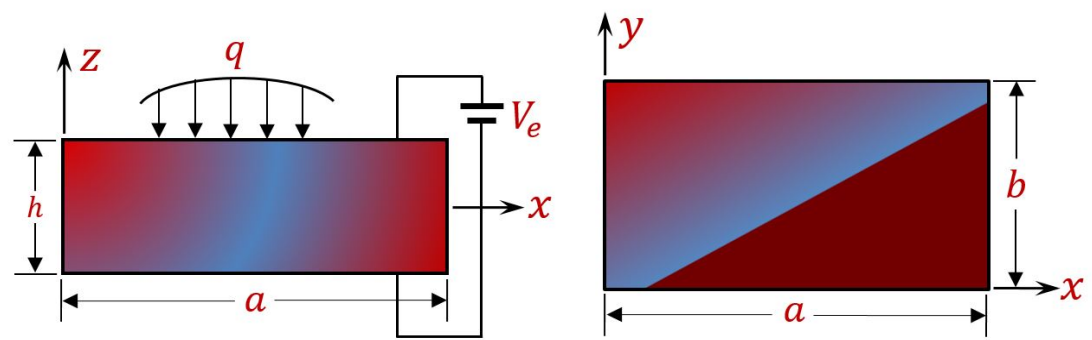

Figure 1: Coordinates and formulation of the FGP plate

\subsection{Quasi-3D sinusoidal theory of FGP plates}

According to the extended quasi-3D sinusoidal plate theory (Zenkour 2007), the displacement components and electric potential of such theory are given as:

$$
\begin{gathered}
u_{1}(x, y, z)=u(x, y)-z \frac{\partial w(x, y)}{\partial x}+\frac{1}{\xi} \sin (\xi z) \psi_{1}(x, y), \\
u_{2}(x, y, z)=v(x, y)-z \frac{\partial w(x, y)}{\partial y}+\frac{1}{\xi} \sin (\xi z) \psi_{2}(x, y), \\
u_{3}(x, y, z)=w(x, y)+\cos (\xi z) \psi_{3}(x, y), \\
\Phi(x, z, t)=-\cos (\xi z) \phi(x, t)+\frac{2 z}{h} V_{e}, \xi=\frac{\pi}{h},
\end{gathered}
$$

where $u, v$ and $w$ are the displacements of mid-plane along the axes $x, y$ and $z$ respectively, $\psi_{1}, \psi_{2}$ denote rotational displacement about $y, x$ axes, respectively, $\psi_{3}$ is additional displacement to show the inclusion of normal deformation, and $\phi(x, t)$ is the electric potential on the mid-plane. The displacement component $w(x, y)$ of the current theory is select based on the following assumptions: the axial in-plane and transverse displacements are partitioned into bending $w_{b}$ and shear $w_{s}$ components as 
$w(x, y)=w_{b}(x, y)+w_{s}(x, y)$

the bending component likes to that given by the CPT; while the shear component gives rise to the sinusoidal variations of shear strains and hence to shear stresses through the thickness of the plate, in such a way that shear stresses are vanish on the top and bottom surfaces of the plate.

Then, the displacement field can be obtained by using Eq. (3) in the form:

$$
\begin{gathered}
u_{1}(x, y, z)=u(x, y)-z \frac{\partial w_{b}}{\partial x}-f(z) \frac{\partial w_{s}}{\partial x}, \\
u_{2}(x, y, z)=v(x, y)-z \frac{\partial w_{b}}{\partial y}-f(z) \frac{\partial w_{s}}{\partial y}, \\
u_{3}(x, y, z)=w_{b}(x, y)+w_{s}(x, y)+g(z) \psi_{3}(x, y), \\
\Phi(x, z, t)=-g(z) \phi(x, t)+\frac{2 z}{h} V_{e},
\end{gathered}
$$

where $f(z)=z-\frac{1}{\xi} \sin (\xi z)$ and $g(z)=1-f^{\prime}(z)=\cos (\xi z)$. Based on Eq. (5), the number of unknown functions is only six. The linear strains with the displacements in Eq. (5) are

$$
\begin{gathered}
\varepsilon_{i i}=\varepsilon_{i i}^{0}-z \varepsilon_{i i}^{1}-f(z) \varepsilon_{i i}^{2}, \quad i=1,2 \\
\gamma_{12}=\gamma_{12}^{0}+z \gamma_{12}^{1}+f(z) \gamma_{12}^{2} \\
\varepsilon_{33}=g^{\prime}(z) \varepsilon_{33}^{0}, \quad \gamma_{i 3}=g(z) \gamma_{i 3}^{0}
\end{gathered}
$$

where

$$
\begin{gathered}
\varepsilon_{11}^{0}=\frac{\partial u}{\partial x}, \quad \varepsilon_{11}^{1}=\frac{\partial^{2} w_{b}}{\partial x^{2}}, \quad \varepsilon_{11}^{2}=\frac{\partial^{2} w_{s}}{\partial x^{2}}, \quad \varepsilon_{22}^{0}=\frac{\partial v}{\partial y}, \quad \varepsilon_{22}^{1}=\frac{\partial^{2} w_{b}}{\partial y^{2}}, \\
\varepsilon_{22}^{2}=\frac{\partial^{2} w_{s}}{\partial y^{2}}, \quad \varepsilon_{33}^{0}=\psi_{3}, \quad \gamma_{12}^{0}=\frac{\partial u}{\partial y}+\frac{\partial v}{\partial x}, \quad \gamma_{12}^{1}=-2 \frac{\partial^{2} w_{b}}{\partial x \partial y} \\
\gamma_{12}^{2}=-2 \frac{\partial^{2} w_{s}}{\partial x \partial y}, \quad \gamma_{23}^{0}=\frac{\partial w_{s}}{\partial y}+\frac{\partial \psi_{3}}{\partial y}, \quad \gamma_{13}^{0}=\frac{\partial w_{s}}{\partial x}+\frac{\partial \psi_{3}}{\partial x} .
\end{gathered}
$$

Coupled elastic and electrical field equations due to (Tiersten 1969) are given as

$$
\begin{aligned}
\sigma_{i j} & =c_{i j k l} \varepsilon_{k l}-e_{k i j} E_{k}, \\
D_{i} & =e_{i k l} \varepsilon_{k l}+\mu_{i k} E_{k},
\end{aligned}
$$

where $\sigma_{i j}, D_{i}, \varepsilon_{i j}$ and $E_{i}$ are the stress tensors, electric displacement vector, strain and electric field components, respectively; $c_{i j k l}, e_{k i j}$ and $\mu_{i k}$ are elastic, piezoelectric and dielectric coefficients, respectively.

The fourth-order stiffness tensor $c_{i j k l}$ may be written as a tensor of second order (as symmetric matrix $c_{N M}$ ) with $11 \rightarrow 1 ; 22 \rightarrow 2 ; 33 \rightarrow 3 ; 23,32 \rightarrow 4 ; 13,31 \rightarrow 5 ;$ and $12,21 \rightarrow 6$. The corresponding stress-strain relations accounting for piezoelectric and thermal effects can be written as:

$$
\begin{aligned}
& \left\{\begin{array}{l}
\sigma_{11} \\
\sigma_{22} \\
\sigma_{33} \\
\sigma_{12}
\end{array}\right\}=\left[\begin{array}{cccc}
c_{11} & c_{12} & c_{13} & 0 \\
c_{12} & c_{22} & c_{23} & 0 \\
c_{13} & c_{23} & c_{33} & 0 \\
0 & 0 & 0 & c_{66}
\end{array}\right]\left\{\begin{array}{l}
\varepsilon_{11}-\alpha(z) \Delta T-\beta(z) \Delta C \\
\varepsilon_{22}-\alpha(z) \Delta T-\beta(z) \Delta C \\
\varepsilon_{33}-\alpha(z) \Delta T-\beta(z) \Delta C \\
\gamma_{12}-\alpha(z) \Delta T-\beta(z) \Delta C
\end{array}\right\}-\left[\begin{array}{ccc}
0 & 0 & e_{31} \\
0 & 0 & e_{32} \\
0 & 0 & e_{33} \\
0 & 0 & 0
\end{array}\right]\left\{\begin{array}{l}
E_{1} \\
E_{2} \\
E_{3}
\end{array}\right\}, \\
& \left\{\begin{array}{l}
\sigma_{23} \\
\sigma_{13}
\end{array}\right\}=\left[\begin{array}{cc}
c_{44} & 0 \\
0 & c_{55}
\end{array}\right]\left\{\begin{array}{l}
\gamma_{23} \\
\gamma_{13}
\end{array}\right\}-\left[\begin{array}{ccc}
0 & e_{24} & 0 \\
e_{15} & 0 & 0
\end{array}\right]\left\{\begin{array}{l}
E_{1} \\
E_{2} \\
E_{3}
\end{array}\right\},
\end{aligned}
$$


$\left\{\begin{array}{l}D_{1} \\ D_{2} \\ D_{3}\end{array}\right\}=\left[\begin{array}{cccccc}0 & 0 & 0 & 0 & e_{15} & 0 \\ 0 & 0 & 0 & e_{24} & 0 & 0 \\ e_{31} & e_{32} & e_{33} & 0 & 0 & 0\end{array}\right]\left\{\begin{array}{c}\varepsilon_{11}-\alpha(z) \Delta T-\beta(z) \Delta C \\ \varepsilon_{22}-\alpha(z) \Delta T-\beta(z) \Delta C \\ \varepsilon_{33}-\alpha(z) \Delta T-\beta(z) \Delta C \\ \gamma_{23} \\ \gamma_{13} \\ \gamma_{12}-\alpha(z) \Delta T-\beta(z) \Delta C\end{array}\right\}+\left[\begin{array}{ccc}\mu_{11} & 0 & 0 \\ 0 & \mu_{22} & 0 \\ 0 & 0 & \mu_{33}\end{array}\right]\left\{\begin{array}{l}E_{1} \\ E_{2} \\ E_{3}\end{array}\right\}$

The non-zero components of electric field $\left(E_{1}, E_{2}, E_{3}\right)$ are defined as:

$\left\{\begin{array}{l}E_{1} \\ E_{2} \\ E_{3}\end{array}\right\}=-\left\{\begin{array}{l}\frac{\partial \Phi}{\partial x} \\ \frac{\partial \Phi}{\partial y} \\ \frac{\partial \Phi}{\partial z}\end{array}\right\}=\left\{\begin{array}{c}\cos (\xi z) \frac{\partial \phi}{\partial x} \\ \cos (\xi z) \frac{\partial \phi}{\partial y} \\ -\xi \sin (\xi z) \phi-\frac{2}{h} V_{e}\end{array}\right\}$

The generalized temperature field variation and moisture exposure through the FGP plate is assumed as

$\left\{\begin{array}{l}T(x, y, z) \\ C(x, y, z)\end{array}\right\}=\left\{\begin{array}{l}T_{1}(x, y) \\ C_{1}(x, y)\end{array}\right\}+\frac{z}{h}\left\{\begin{array}{l}T_{2}(x, y) \\ C_{2}(x, y)\end{array}\right\}+\frac{f(z)}{h}\left\{\begin{array}{l}T_{3}(x, y) \\ C_{3}(x, y)\end{array}\right\}$

where $T_{i}$ and $C_{i}$ are the thermal and moisture loads.

\section{EQUILIBRIUM EQUATIONS AND BOUNDARY CONDITIONS}

\subsection{General formulations}

The principle of virtual work is stated as

$\int_{\Omega} \int_{-h / 2}^{h / 2}\left(\sigma_{11} \delta \varepsilon_{11}+\sigma_{22} \delta \varepsilon_{22}+\sigma_{33} \delta \varepsilon_{33}+\sigma_{23} \delta \gamma_{23}+\sigma_{13} \delta \gamma_{13}+\sigma_{12} \delta \gamma_{12}\right) \mathrm{d} z \mathrm{~d} \Omega$

$-\int_{\Omega} \int_{-h / 2}^{h / 2}\left(D_{1} \delta E_{1}+D_{2} \delta E_{2}+D_{3} \delta E_{3}\right) \mathrm{d} z \mathrm{~d} \Omega-\int_{\Omega} q\left(w_{b}+w_{s}+g( \pm h / 2) \psi_{3}\right) \mathrm{d} \Omega=0$,

where $q$ is the sinusoidally distributed load of the plate. Substituting Eqs. (6), (7) and (10) into Eq. (12) gives the equilibrium equations in the form

$\delta u: \frac{\partial N_{11}}{\partial x}+\frac{\partial N_{12}}{\partial y}=0, \quad \delta v: \frac{\partial N_{12}}{\partial x}+\frac{\partial N_{22}}{\partial y}=0$,

$\delta w_{b}: \frac{\partial^{2} M_{11}}{\partial x^{2}}+2 \frac{\partial^{2} M_{12}}{\partial x \partial y}+\frac{\partial^{2} M_{22}}{\partial y^{2}}+q=0$,

$\delta w_{s}: \frac{\partial^{2} P_{11}}{\partial x^{2}}+2 \frac{\partial^{2} P_{12}}{\partial x \partial y}+\frac{\partial^{2} P_{22}}{\partial y^{2}}+\frac{\partial Q_{1}}{\partial x}+\frac{\partial Q_{2}}{\partial y}+q=0$,

$\delta \psi_{3}: \frac{\partial Q_{1}}{\partial x}+\frac{\partial Q_{2}}{\partial y}-N_{33}=0$,

$\delta \phi: \int_{-h / 2}^{h / 2}\left(g(z) \frac{\partial D_{1}}{\partial x}+g(z) \frac{\partial D_{2}}{\partial y}+g^{\prime}(z) D_{3}\right) \mathrm{d} z$,

where the resultant components are defined as 


$$
\begin{aligned}
& \left\{N_{i j}, M_{i j}, P_{i j}\right\}=\int_{-h / 2}^{h / 2} \sigma_{i j}\{1, z, f(z)\} \mathrm{d} z, i, j=1,2, \\
& \left\{Q_{i}, N_{33}\right\}=\int_{-h / 2}^{h / 2}\left\{g(z) \sigma_{i 3}, g^{\prime}(z) \sigma_{33}\right\} \mathrm{d} z, i=1,2,
\end{aligned}
$$

in which $N_{i j}$ and $M_{i j}$ are the stress resultants and stress couples, $P_{i j}$ are additional higher-order stress couples given according to $f(z)$, and $Q_{i}$ and $N_{33}$ are the transverse and normal shear stress resultants.

Also, the boundary conditions may be expressed as

$\delta u: N_{11} n_{1}+N_{12} n_{2}=0, \delta v: N_{12} n_{1}+N_{22} n_{2}=0$,

$\delta w_{b}:\left(\frac{\partial M_{11}}{\partial x}+\frac{\partial M_{12}}{\partial y}\right) n_{1}+\left(\frac{\partial M_{12}}{\partial x}+\frac{\partial M_{22}}{\partial y}\right) n_{2}+\frac{\partial}{\partial r}\left[\left(M_{22}-M_{11}\right) n_{1} n_{2}+M_{12}\left(n_{1}^{2}-n_{2}^{2}\right)\right]=0$,

$\delta w_{S}:\left(Q_{1}+\frac{\partial P_{11}}{\partial x}+\frac{\partial P_{12}}{\partial y}\right) n_{1}+\left(Q_{2}+\frac{\partial P_{12}}{\partial x}+\frac{\partial P_{22}}{\partial y}\right) n_{2}$

$$
+\frac{\partial}{\partial r}\left[\left(P_{22}-P_{11}\right) n_{1} n_{2}+P_{12}\left(n_{1}^{2}-n_{2}^{2}\right)\right]=0,
$$

$\delta \psi_{3}: Q_{1} n_{1}+Q_{2} n_{2}, \frac{\partial \delta w_{b}}{\partial m}: M_{11} n_{1}^{2}+M_{22} n_{2}^{2}+2 M_{12} n_{1} n_{2}=0$,

$\frac{\partial \delta w_{s}}{\partial m}: P_{11} n_{1}^{2}+P_{22} n_{2}^{2}+2 P_{12} n_{1} n_{2}=0$,

$\delta \phi: \int_{-h / 2}^{h / 2}\left(D_{1} n_{1}+D_{2} n_{2}\right) g(z) \mathrm{d} z=0$,

where

$\frac{\partial}{\partial r}=\frac{\partial}{\partial y} n_{1}-\frac{\partial}{\partial x} n_{2}, \quad \frac{\partial}{\partial m}=\frac{\partial}{\partial x} n_{1}+\frac{\partial}{\partial y} n_{2}$.

According to Eq. (14), the expressions of resultant components are given by

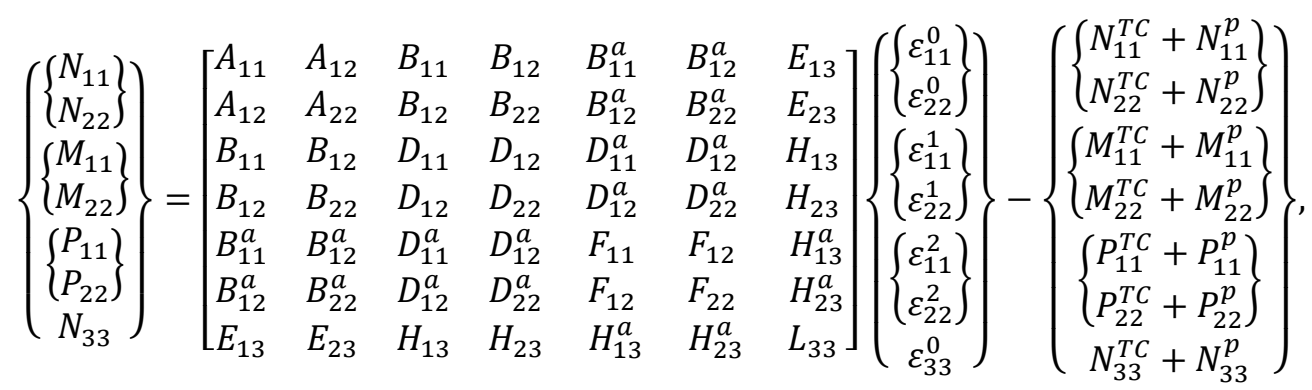

$\left\{\begin{array}{c}N_{12} \\ M_{12} \\ P_{12}\end{array}\right\}=\left[\begin{array}{lll}A_{66} & B_{66} & B_{66}^{a} \\ B_{66} & D_{66} & D_{66}^{a} \\ B_{66}^{a} & D_{66}^{a} & F_{66}\end{array}\right]\left\{\begin{array}{c}\gamma_{12}^{0} \\ \gamma_{12}^{1} \\ \gamma_{12}^{2}\end{array}\right\}-\left\{\begin{array}{c}N_{12}^{T C} \\ M_{12}^{T C} \\ P_{12}^{T C}\end{array}\right\}$,

$\left\{\begin{array}{l}Q_{2} \\ Q_{1}\end{array}\right\}=\left[\begin{array}{cc}A_{44}^{a} & 0 \\ 0 & A_{55}^{a}\end{array}\right]\left\{\begin{array}{l}\gamma_{23}^{0} \\ \gamma_{13}^{0}\end{array}\right\}-\left\{\begin{array}{l}Q_{2}^{p} \\ Q_{1}^{p}\end{array}\right\}$,

where the stiffness parameters are expressed as 


$$
\begin{gathered}
\left\{A_{i j}, B_{i j}, B_{i j}^{a}, D_{i j}, D_{i j}^{a}, F_{i j}\right\}=\int_{-h / 2}^{h / 2} c_{i j}\left\{1, z, f, z^{2}, f z, f^{2}\right\} \mathrm{d} z,(i, j=1,2), \\
\left\{E_{i 3}, H_{i 3}, H_{i 3}^{a}\right\}=\int_{-h / 2}^{h / 2} c_{i 3}\left\{g^{\prime}, g^{\prime} z, g^{\prime} f\right\} \mathrm{d} z,(i=1,2), \\
A_{i i}^{a}=\int_{-h / 2}^{h / 2} c_{i i} g^{2} \mathrm{~d} z,(i=4,5), L_{33}=\int_{-h / 2}^{h / 2} c_{33} g^{\prime 2} \mathrm{~d} z .
\end{gathered}
$$

Also, the resultant components, due to hygro-thermal loadings, are given by:

$$
\begin{gathered}
\left\{N_{i i}^{T C}, M_{i i}^{T C}, P_{i i}^{T C}\right\}=\int_{-h / 2}^{h / 2}\left(c_{1 i}+c_{i 2}+c_{i 3}\right)(\alpha(z) \Delta T+\beta(z) \Delta C)\{1, z, f(z)\} \mathrm{d} z, i=1,2, \\
\left\{N_{12}^{T C}, M_{12}^{T C}, P_{12}^{T C}\right\}=\int_{-h / 2}^{h / 2} c_{66}(\alpha(z) \Delta T+\beta(z) \Delta C)\{1, z, f(z)\} \mathrm{d} z \\
\left\{N_{33}^{T C}\right\}=\int_{-h / 2}^{h / 2}\left(c_{13}+c_{23}+c_{33}\right)(\alpha(z) \Delta T+\beta(z) \Delta C) g^{\prime}(z) \mathrm{d} z \\
\left\{D_{3}^{T C}\right\}=\int_{-h / 2}^{h / 2}\left(e_{31}+e_{32}+e_{33}\right)(\alpha(z) \Delta T+\beta(z) \Delta C) g^{\prime}(z) \mathrm{d} z
\end{gathered}
$$

Finally, the electrical moments and stress resultants are given by

$\left\{N_{i i}^{p}, M_{i i}^{p}, P_{i i}^{p}\right\}=\int_{-h / 2}^{h / 2} e_{3 i} E_{3}\{1, z, f(z)\} \mathrm{d} z, i=1,2$,

$\left\{\begin{array}{l}N_{33}^{p} \\ Q_{2}^{p} \\ Q_{1}^{p}\end{array}\right\}=\int_{-h / 2}^{h / 2}\left[\begin{array}{ccc}0 & 0 & e_{33} \\ 0 & e_{24} & 0 \\ e_{15} & 0 & 0\end{array}\right]\left\{\begin{array}{l}E_{1} \\ E_{2} \\ E_{3}\end{array}\right\} \mathrm{d} z$.

Finally, substituting Eqs. (9) and (16) into Eq. (13) gives the governing equations as

$$
\begin{aligned}
& A_{11} \frac{\partial^{2} u}{\partial x^{2}}+A_{66} \frac{\partial^{2} u}{\partial y^{2}}+\left(A_{12}+A_{66}\right) \frac{\partial^{2} v}{\partial x \partial y}-B_{11} \frac{\partial^{3} w_{b}}{\partial x^{3}}-\left(B_{12}+2 B_{66}\right) \frac{\partial^{3} w_{b}}{\partial x \partial y^{2}}-B_{11}^{a} \frac{\partial^{3} w_{s}}{\partial x^{3}} \\
& -\left(B_{12}^{a}+2 B_{66}^{a}\right) \frac{\partial^{3} w_{s}}{\partial x \partial y^{2}}+E_{13} \frac{\partial \psi_{3}}{\partial x}+\bar{A}_{31}^{p} \frac{\partial \phi}{\partial x}=f_{1},
\end{aligned}
$$

$A_{66} \frac{\partial^{2} v}{\partial x^{2}}+A_{22} \frac{\partial^{2} v}{\partial y^{2}}+\left(A_{12}+A_{66}\right) \frac{\partial^{2} u}{\partial x \partial y}-B_{22} \frac{\partial^{3} w_{b}}{\partial y^{3}}-\left(B_{12}+2 B_{66}\right) \frac{\partial^{3} w_{b}}{\partial x^{2} \partial y}$

$-B_{22}^{a} \frac{\partial^{3} w_{s}}{\partial y^{3}}-\left(B_{12}^{a}+2 B_{66}^{a}\right) \frac{\partial^{3} w_{s}}{\partial x^{2} \partial y}+E_{23} \frac{\partial \psi_{3}}{\partial x}+\vec{A}_{31}^{p} \frac{\partial \phi}{\partial y}=f_{2}$,

$B_{11} \frac{\partial^{3} u}{\partial x^{3}}+\left(B_{12}+2 B_{66}\right)\left(\frac{\partial^{3} u}{\partial x \partial y^{2}}+\frac{\partial^{3} v}{\partial x^{2} \partial y}\right)+B_{22} \frac{\partial^{3} v}{\partial y^{3}}-D_{11} \frac{\partial^{4} w_{b}}{\partial x^{4}}-D_{22} \frac{\partial^{4} w_{b}}{\partial y^{4}}$

$-2\left(D_{12}+2 D_{66}\right) \frac{\partial^{4} w_{b}}{\partial x^{2} \partial y^{2}}-D_{11}^{a} \frac{\partial^{4} w_{s}}{\partial x^{4}}-D_{22}^{a} \frac{\partial^{4} w_{s}}{\partial y^{4}}-2\left(D_{12}^{a}+2 D_{66}^{a}\right) \frac{\partial^{4} w_{s}}{\partial x^{2} \partial y^{2}}$

$+H_{13} \frac{\partial^{2} \psi_{3}}{\partial x^{2}}+H_{23} \frac{\partial^{2} \psi_{3}}{\partial y^{2}}+\bar{A}_{31}^{z p} \frac{\partial^{2} \phi}{\partial x^{2}}+\bar{A}_{31}^{z p} \frac{\partial^{2} \phi}{\partial y^{2}}=f_{3}$,

$B_{11}^{a} \frac{\partial^{3} u}{\partial x^{3}}+\left(B_{12}^{a}+2 B_{66}^{a}\right)\left(\frac{\partial^{3} u}{\partial x \partial y^{2}}+\frac{\partial^{3} v}{\partial x^{2} \partial y}\right)+B_{22}^{a} \frac{\partial^{3} v}{\partial y^{3}}-D_{11}^{a} \frac{\partial^{4} w_{b}}{\partial x^{4}}-D_{22}^{a} \frac{\partial^{4} w_{b}}{\partial y^{4}}$

$-2\left(D_{12}^{a}+2 D_{66}^{a}\right) \frac{\partial^{4} w_{b}}{\partial x^{2} \partial y^{2}}-F_{11} \frac{\partial^{4} w_{s}}{\partial x^{4}}-F_{22} \frac{\partial^{4} w_{s}}{\partial y^{4}}-2\left(F_{12}+2 F_{66}\right) \frac{\partial^{4} w_{s}}{\partial x^{2} \partial y^{2}}$

$+A_{55}^{a} \frac{\partial^{2} w_{s}}{\partial x^{2}}+A_{44}^{a} \frac{\partial^{2} w_{s}}{\partial y^{2}}+\left(H_{13}^{a}+A_{55}^{a}\right) \frac{\partial^{2} \psi_{3}}{\partial x^{2}}+\left(H_{23}^{a}+A_{44}^{a}\right) \frac{\partial^{2} \psi_{3}}{\partial y^{2}}$ 
$-E_{13} \frac{\partial u}{\partial x}-E_{23} \frac{\partial v}{\partial y}+H_{13} \frac{\partial^{2} w_{b}}{\partial x^{2}}+H_{23} \frac{\partial^{2} w_{b}}{\partial y^{2}}+\left(H_{13}^{a}+A_{55}^{a}\right) \frac{\partial^{2} w_{s}}{\partial x^{2}}+\left(H_{23}^{a}+A_{44}^{a}\right) \frac{\partial^{2} w_{s}}{\partial y^{2}}$

$+A_{55}^{a} \frac{\partial^{2} \psi_{3}}{\partial x^{2}}+A_{44}^{a} \frac{\partial^{2} \psi_{3}}{\partial y^{2}}-L_{33} \psi_{3}-\bar{A}_{24}^{p} \frac{\partial^{2} \phi}{\partial y^{2}}-\bar{A}_{15}^{p} \frac{\partial^{2} \phi}{\partial x^{2}}=f_{5}$,

$\bar{A}_{15}^{p} \frac{\partial^{2} w_{s}}{\partial x^{2}}+\bar{A}_{15}^{p} \frac{\partial^{2} \psi_{3}}{\partial x^{2}}+\bar{D}_{11}^{p} \frac{\partial^{2} \phi}{\partial x^{2}}+\bar{A}_{24}^{p} \frac{\partial^{2} w_{s}}{\partial y^{2}}+\bar{A}_{24}^{p} \frac{\partial^{2} \psi_{3}}{\partial y^{2}}+\bar{D}_{11}^{p} \frac{\partial^{2} \phi}{\partial y^{2}}+\bar{A}_{31}^{p}\left(\frac{\partial u}{\partial x}+\frac{\partial v}{\partial y}\right)$

$-\bar{A}_{31}^{z p}\left(\frac{\partial^{2} w_{b}}{\partial x^{2}}+\frac{\partial^{2} w_{b}}{\partial y^{2}}\right)-\bar{A}_{31}^{f p}\left(\frac{\partial^{2} w_{s}}{\partial x^{2}}+\frac{\partial^{2} w_{s}}{\partial x^{2}}\right)+\bar{A}_{33}^{p} \psi_{3}-\bar{H}_{33}^{p} \phi-\bar{H}_{33}^{p p} V_{e}=f_{6}$,

where

$\left\{\bar{A}_{31}^{p}, \bar{A}_{31}^{z p}, \bar{A}_{31}^{f p}\right\}=\int_{-h / 2}^{h / 2} e_{31}\{1, z, f(z)\} \xi \sin (\xi z) \mathrm{d} z$

$\bar{A}_{i j}^{p}=\int_{-h / 2}^{h / 2} e_{i j} \cos ^{2}(\xi z) \mathrm{d} z$

$\bar{D}_{11}^{p}=\int_{-h / 2}^{h / 2} \mu_{11} \cos ^{2}(\xi z) \mathrm{d} z$

$\left\{\bar{H}_{33}^{p}, \bar{H}_{33}^{p p}\right\}=\int_{-h / 2}^{h / 2}\left\{\mu_{11} \xi^{2} \sin ^{2}(\xi z), \mu_{11} \frac{2}{h} \xi \sin (\xi z)\right\} \mathrm{d} z$.

Also, the force components $f_{i}$ are given by

$f_{1}=\frac{\partial N_{11}^{T C}}{\partial x}+\frac{\partial N_{12}^{T C}}{\partial y}, \quad f_{2}=\frac{\partial N_{12}^{T C}}{\partial x}+\frac{\partial N_{22}^{T C}}{\partial y}$

$f_{3}=-q+\frac{\partial^{2} M_{11}^{T C}}{\partial x^{2}}+2 \frac{\partial^{2} M_{12}^{T C}}{\partial x \partial y}+\frac{\partial^{2} M_{22}^{T C}}{\partial y^{2}}$,

$f_{4}=-q+\frac{\partial^{2} P_{11}^{T C}}{\partial x^{2}}+2 \frac{\partial^{2} P_{12}^{T C}}{\partial x \partial y}+\frac{\partial^{2} P_{22}^{T C}}{\partial y^{2}}, \quad f_{5}=N_{33}^{T C}, \quad f_{6}=D_{3}^{T C}$.

\section{CLOSED-FORM SOLUTION}

We assume that the EGP or FGP rectangular plate is simply-supported with its four edges, therefore, the boundary conditions of the plates are assumed to be

$u(x, 0)=u(x, b)=v(0, y)=v(a, y)=0$,

$u(x, 0)=u(x, b)=v(0, y)=v(a, y)=0$,

$w_{b}(x, 0)=w_{b}(x, b)=w_{b}(0, y)=w_{b}(a, y)=0$,

$w_{S}(x, 0)=w_{s}(x, b)=w_{s}(0, y)=w_{s}(a, y)=0$,

$\psi_{3}(x, 0)=\psi_{3}(x, b)=\psi_{3}(0, y)=\psi_{3}(a, y)=0$.

So, the complete solution to the governing equations is expressed as 
$\left\{\begin{array}{c}u \\ v \\ {\left[w_{b}, w_{s}, \psi_{3}, \phi\right]}\end{array}\right\}=\left\{\begin{array}{c}U \cos (\lambda x) \sin (\mu y) \\ V \sin (\lambda x) \cos (\mu y) \\ {\left[W_{b}, W_{s}, Z, Y\right] \sin (\alpha x) \sin (\beta y)}\end{array}\right\}$,

where $\lambda=\pi / a, \mu=\pi / b$, and $U, V, W_{b}, W_{s}, Z$ and $\gamma$ are the unknown Fourier expansion coefficients. Since a sinusoidally distributed load is applied on the upper surface of the plate, so the form of the distributed load as well as the transverse hygrothermal $T_{i}$ and $C_{i}$ loads is given in the form

$\left\{\begin{array}{l}q(x, y) \\ T_{i}(x, y) \\ C_{i}(x, y)\end{array}\right\}=\left\{\begin{array}{l}q_{0} \\ \bar{T}_{i} \\ \bar{C}_{i}\end{array}\right\} \sin (\lambda x) \sin (\mu y),(i=1,2,3)$.

Substituting Eqs. (22), (23) into Eq. (19), one obtains the following operator equation:

$[K]\{\Delta\}=\{F\}$,

where $\{\Delta\}=\left\{U, V, W_{b}, W_{s}, Z, Y\right\}^{t},\{F\}=\left\{F_{1}, F_{2}, F_{3}, F_{4}, F_{5}, F_{6}\right\}^{t}$ and the coefficients of the symmetric stiffness matrix $k_{i j}=k_{j i}$ are given by

$k_{11}=A_{11} \lambda^{2}+A_{66} \mu^{2}, k_{12}=\left(A_{11}+A_{66}\right) \lambda \mu$,

$k_{13}=-B_{11} \lambda^{3}-\left(B_{12}+2 B_{66}\right) \lambda \mu^{2}, k_{15}=-E_{13} \lambda$,

$k_{14}=-B_{11}^{a} \lambda^{3}-\left(B_{12}^{a}+2 B_{66}^{a}\right) \lambda \mu^{2}, k_{16}=\bar{A}_{31}^{p} \lambda$,

$k_{22}=A_{66} \lambda^{2}+A_{22} \mu^{2}, k_{23}=-B_{22} \mu^{3}-\left(B_{12}+2 B_{66}\right) \lambda^{2} \mu$,

$k_{24}=-B_{22}^{a} \mu^{3}-\left(B_{12}^{a}+2 B_{66}^{a}\right) \lambda^{2} \mu, k_{25}=-E_{23} \mu, k_{26}=\bar{A}_{31}^{p} \lambda$,

$k_{33}=D_{11} \lambda^{4}+2\left(D_{12}+2 D_{66}\right) \lambda^{2} \mu^{2}+D_{22} \mu^{4}, k_{35}=H_{13} \lambda^{2}+H_{23} \mu^{2}$,

$k_{34}=D_{11}^{a} \lambda^{4}+2\left(D_{12}^{a}+2 D_{66}^{a}\right) \lambda^{2} \mu^{2}+D_{22}^{a} \mu^{4}, k_{36}=-\bar{A}_{31}^{z p}\left(\lambda^{2}+\mu^{2}\right)$,

$k_{45}=\left(H_{13}^{a}+A_{55}^{a}\right) \lambda^{2}+\left(H_{23}^{a}+A_{44}^{a}\right) \mu^{2}, k_{55}=A_{55}^{a} \lambda^{2}+A_{44}^{a} \mu^{2}+L_{33}$,

$k_{44}=F_{11} \lambda^{4}+2\left(F_{12}+2 F_{66}\right) \lambda^{2} \mu^{2}+F_{22} \mu^{4}+A_{55}^{a} \lambda^{2}+A_{44}^{a} \mu^{2}$,

$k_{46}=\bar{A}_{15}^{p} \lambda^{2}+\bar{A}_{24}^{p} \mu^{2}-\bar{A}_{31}^{f p}\left(\lambda^{2}+\mu^{2}\right)$,

$k_{56}=\bar{A}_{15}^{p} \lambda^{2}+\bar{A}_{24}^{p} \mu^{2}, k_{66}=\bar{D}_{11}^{p}\left(\lambda^{2}+\mu^{2}\right)+\bar{H}_{33}^{p}$,

in which that components of the generalized force vector are given by

$F_{1}=\lambda\left(A_{1}^{*} \bar{T}_{1}+B_{1}^{*} \bar{T}_{2}^{*}+D_{1}^{*} \bar{T}_{3}^{*}+a_{1}^{*} \bar{H}_{1}+b_{1}^{*} \bar{H}_{2}^{*}+d_{1}^{*} \bar{H}_{3}^{*}\right)$,

$F_{2}=\mu\left(A_{2}^{*} \bar{T}_{1}+B_{2}^{*} \bar{T}_{2}^{*}+D_{2}^{*} \bar{T}_{3}^{*}+a_{2}^{*} \bar{H}_{1}+b_{2}^{*} \bar{H}_{2}^{*}+d_{2}^{*} \bar{H}_{3}^{*}\right)$,

$F_{3}=-q_{0}+\lambda^{2}\left(B_{1}^{*} \bar{T}_{1}+G_{1}^{*} \bar{T}_{2}^{*}+F_{1}^{*} \bar{T}_{3}^{*}+b_{1}^{*} \bar{H}_{1}+g_{1}^{*} \bar{H}_{2}^{*}+f_{1}^{*} \bar{H}_{3}^{*}\right)$

$+\mu^{2}\left(B_{2}^{*} \bar{T}_{1}+G_{2}^{*} \bar{T}_{2}^{*}+F_{2}^{*} \bar{T}_{3}^{*}+b_{2}^{*} \bar{H}_{1}+g_{2}^{*} \bar{H}_{2}^{*}+f_{2}^{*} \bar{H}_{3}^{*}\right)$,

$F_{4}=-q_{0}+\lambda^{2}\left(D_{1}^{*} \bar{T}_{1}+F_{1}^{*} \bar{T}_{2}^{*}+E_{1}^{*} \bar{T}_{3}^{*}+d_{1}^{*} \bar{H}_{1}+f_{1}^{*} \bar{H}_{2}^{*}+e_{1}^{*} \bar{H}_{3}^{*}\right)$ 
$+\mu^{2}\left(D_{2}^{*} \bar{T}_{1}+F_{2}^{*} \bar{T}_{2}^{*}+E_{2}^{*} \bar{T}_{3}^{*}+d_{2}^{*} \bar{H}_{1}+f_{2}^{*} \bar{H}_{2}^{*}+e_{2}^{*} \bar{H}_{3}^{*}\right)$

$F_{5}=L_{3}^{*} \bar{T}_{1}+\bar{L}_{3}^{*} \bar{T}_{2}^{*}+\overline{\bar{L}}_{3}^{*} \bar{T}_{3}^{*}+l_{3}^{*} \bar{H}_{1}+\bar{l}_{3}^{*} \bar{H}_{2}^{*}+\overline{\bar{l}}_{3}^{*} \bar{H}_{3}^{*}$

$F_{6}=\bar{H}_{33}^{p p} V_{e}+\hat{E}^{p} \bar{T}_{1}+\hat{E}^{z p} \bar{T}_{2}^{*}+\hat{E}^{f p} \bar{T}_{3}^{*}+\hat{e}^{p} \bar{H}_{1}+\hat{e}^{z p} \bar{H}_{2}^{*}+\hat{e}^{f p} \bar{H}_{3}^{*}$,

where

$\left\{A_{i}^{*}, B_{i}^{*}, D_{i}^{*}, G_{i}^{*}, F_{i}^{*}, E_{i}^{*}\right\}=\int_{-h / 2}^{h / 2}\left(c_{1 i}+c_{i 2}\right) \alpha(z)\left\{1, z, f, z^{2}, z f, f^{2}\right\} \mathrm{d} z,(i=1,2)$,

$\left\{a_{i}^{*}, b_{i}^{*}, d_{i}^{*}, g_{i}^{*}, f_{i}^{*}, e_{i}^{*}\right\}=\int_{-h / 2}^{h / 2}\left(c_{1 i}+c_{i 2}\right) \beta(z)\left\{1, z, f, z^{2}, z f, f^{2}\right\} \mathrm{d} z,(i=1,2)$,

$\left\{L_{3}^{*}, \bar{L}_{3}^{*}, \bar{L}_{3}^{*}\right\}=\int_{-h / 2}^{h / 2}\left(c_{13}+c_{23}\right) \alpha(z) g^{\prime}(z)\{1, z, f\} \mathrm{d} z$,

$\left\{l_{3}^{*}, \bar{l}_{3}^{*}, \bar{l}_{3}^{*}\right\}=\int_{-h / 2}^{h / 2}\left(c_{13}+c_{23}\right) \beta(z) g^{\prime}(z)\{1, z, f\} \mathrm{d} z$,

$\left\{\widehat{E}^{p}, \hat{E}^{z p}, \hat{E}^{f p}\right\}=\int_{-h / 2}^{h / 2}\left(e_{31}+e_{32}+e_{33}\right) \alpha(z) g^{\prime}(z)\{1, z, f\} \mathrm{d} z$,

$\left\{\hat{e}^{p}, \hat{e}^{z p}, \hat{e}^{f p}\right\}=\int_{-h / 2}^{h / 2}\left(e_{31}+e_{32}+e_{33}\right) \beta(z) g^{\prime}(z)\{1, z, f\} \mathrm{d} z$,

$\bar{T}_{i}^{*}=\frac{\bar{T}_{i}}{h}, \quad \bar{H}_{i}^{*}=\frac{\bar{H}_{i}}{h}, \quad(i=2,3)$.

\section{NUMERICAL RESULTS AND DISCUSSIONS}

\subsection{Dimensionless quantities}

In this section, the numerical results for the effect of hygrothermal and mechanical loading on the EGP and FGP plates by using quasi-3D sinusoidal plate theory are thoroughly discussed. The top surface of the plates is ceramic-rich while the bottom surface of the plate is metal-rich. The elastic coefficients $c_{i j}$ for the present plate are given by

$c_{11}=c_{22}=c_{33}=\frac{E(z)}{1-v^{2}}, \quad c_{12}=c_{13}=c_{23}=\frac{v E(z)}{1-v^{2}}, \quad c_{i i}=\frac{E(z)}{2(1+v)}, \quad(i=4,5,6)$.

Now, the EGP consisted of Aluminum/Alumina is considered. Young's modulus for aluminum is $70 \mathrm{GPa}$ while for alumina is $380 \mathrm{GPa}$. Also, Poisson's ratio for both equals 0.3 (Al Khateeb and Zenkour 2014). Also, the FGP plates consisted of Titanium/Zirconia are considered. The material properties are given by (Zidi et al. 2014)

Metal (Titanium, Ti-6Al-4V):

$E_{m}=66.2 \mathrm{GPa}, \quad v=0.3, \quad \alpha_{m}=10.3 \times 10^{-6} \mathrm{C}^{\mathrm{o}}, \quad \beta_{m}=0.33 \mathrm{wt} \% \mathrm{H}_{2} \mathrm{O}$.

Ceramic (Zirconia, $\mathrm{ZrO}_{2}$ ):

$E_{c}=117.0 \mathrm{GPa}, \quad v=0.3, \quad \alpha_{c}=7.11 \times 10^{-6} \mathrm{C}^{\mathrm{o}}, \quad \beta_{c}=0.0 \mathrm{wt} \% \mathrm{H}_{2} \mathrm{O}$.

The non-dimensional form of the deflection and stresses parameters of hygro-thermo-mechanical bending are 
$\bar{W}=\frac{10^{2} D}{q_{0} a^{4}} w, \quad \bar{\phi}=\frac{10^{3} e_{15} h}{q_{0} a^{2}} \phi, \quad D=\frac{h^{3} E}{12\left(1-v^{2}\right)}$,

$\bar{\sigma}_{i i}=\frac{h^{2}}{q_{0} a^{2}} \sigma_{i i}, \quad \bar{D}_{i}=\frac{E_{c} h^{2}}{e_{15} q_{0} a^{2}} D_{i}, \quad i=1,2,3$,

$\bar{\sigma}_{12}=\frac{h}{q_{0} a} \sigma_{12}, \quad \bar{\sigma}_{13}=-\frac{h}{q_{0} a} \sigma_{13}, \quad V_{0}=\frac{V_{e}}{h q_{0}}$.

Table 1: Comparison of dimensionless deflection and stresses of EG square plates under sinusoidally load $\left(k=0, \bar{T}_{i}=\bar{C}_{i}=0\right)$.

\begin{tabular}{cccccc}
\hline \hline Methods & \multicolumn{5}{c}{$\boldsymbol{a} / \boldsymbol{h}=\mathbf{1 0}$} \\
\cline { 2 - 6 } & $\overline{\boldsymbol{w}}$ & $\overline{\boldsymbol{\sigma}}_{\mathbf{1 1}}$ & $\overline{\boldsymbol{\sigma}}_{\mathbf{2 2}}$ & $\overline{\boldsymbol{\sigma}}_{\mathbf{1 2}}$ & $\boldsymbol{\sigma}_{\mathbf{3 3}}$ \\
\hline Benyoucef et al. 2010 & 0.2960 & 1.9955 & 1.3121 & 0.7065 & - \\
Thai and Vo 2013 & 0.2960 & 1.9955 & 1.3121 & 0.7065 & - \\
Al-Khateeb and Zenkour 2014 & 0.2936 & 2.0211 & 1.3240 & 0.6932 & 0.1489 \\
Present & 0.2933 & 2.1247 & 1.2905 & 0.6985 & 0.3623 \\
\hline
\end{tabular}

To verify the accuracy and validity of the present technique, results of EGP and FGP plates are compared with the solutions of several theories obtained by new hyperbolic shear deformation theory (HSDT) (Benyoucef et al. 2010), sinusoidal shear deformation theory (SSDT) (Thai and Vo, 2013), a refined four-unknown plate theory RSPT (AI Khateeb and Zenkour 2014) and Q-3D (Zenkour and Hafed 2019). Tables 1-5 show the comparison of dimensionless deflection and stresses of Aluminum/Alumina EGP rectangular plate under sinusoidally load, while Table 6 illustrates the comparison of dimensionless deflection and stresses of Titanium/Zirconia FGP rectangular plate under sinusoidally load.

\subsection{Effect of gradient index}

Some numerical examples for the simply-supported bending of FGP rectangular plate are provided based on quasi3D sinusoidal plate theories. The results of the EG plates given in Table 1 are the same as those of the solution of AlKhateeb and Zenkour (2014), as well as results of the EGP plates in other Tables 2-5. The dimensionless deflection $\bar{w}$ decreases as $k$ increases. The normal stresses $\bar{\sigma}_{11}$ and $\bar{\sigma}_{22}$ decrease as $\mathrm{k}$ decreases, while the transverse shear stress $\bar{\sigma}_{13}$ increases. It is to be noted in Table 6 that the dimensionless deflection $\bar{w}$ decreases as $k$ and $a / h$ increase. The normal stresses $\bar{\sigma}_{11}$ and $\bar{\sigma}_{33}$ increase as $a / h$ and $k$ increase. If we neglect the hygrothermal effect in Table 6 we will get the same results as those in Zenkour and Hafed (2019).

\subsection{Hygro-thermal and piezoelectric effect}

Some further results in Tables 2-5 for EGP plates demonstrate the effect of the external electric voltage $V_{0}$. They are compensated by values as $V_{0}=-0.5,0$ and 0.5 . The deflections $\bar{w}$ in Table 2 at $a / h=2$ and $V_{0}=0$ are very closed of the FGP plates, while in this case the values of deflections decrease as $V_{0}$ and $k$ increase. Tables 3 and 4 show that the dimensionless normal stresses $\bar{\sigma}_{11}, \bar{\sigma}_{22}$ for FGP plates at $a / h=10$ are smaller than the result corresponding ones for FGP plates. In the all tables of FGP rectangular plates, the value of $V_{0}=-0.5$ gives the largest result deflections while $V_{0}=0.5$ gives the smallest ones. In Table 5 , the change of the value of $V_{0}$ does not affect the behavior of shear stresses $\bar{\sigma}_{13}$, also, the shear stresses $\bar{\sigma}_{13}$ for FGP plates are the same as those for FGP plates. In Table 6 , the dimensionless deflection and normal stresses of FGP nonhomogeneous plates, the deflections $\bar{w}$ decrease as $a / h$ increases while $\bar{\sigma}_{11}$, $\bar{\sigma}_{33}$ increase as $a / h$ increases. The effect of the graded $k$ demonstrates the deflections $\bar{w}$ and $\bar{\sigma}_{11}$ decrease as $k$ increases while $\bar{\sigma}_{33}$ increases as $k$ increases.

Table 2: The dimensionless deflection $\bar{w}$ of EGP plates $\left(q_{0}=100, \bar{T}_{1}=\bar{T}_{3}=0, \bar{C}_{1}=\bar{C}_{3}=0, \bar{T}_{2}=100, \bar{C}_{2}=2\right)$.

\begin{tabular}{cccccccccc}
\hline \hline \multirow{2}{*}{$\boldsymbol{a} / \boldsymbol{b}$} & $\boldsymbol{k}$ & \multicolumn{9}{c}{$\boldsymbol{a} / \boldsymbol{h}=\mathbf{2}$} & \multicolumn{3}{c}{$\boldsymbol{a} / \boldsymbol{h}=\mathbf{1 0}$} \\
\cline { 3 - 9 } & & $\mathbf{F G}$ & $\boldsymbol{V}_{\mathbf{0}}=-\mathbf{0 . 5}$ & $\boldsymbol{V}_{\mathbf{0}}=\mathbf{0}$ & $\boldsymbol{V}_{\mathbf{0}}=\mathbf{0 . 5}$ & $\boldsymbol{F G}$ & $\boldsymbol{V}_{\mathbf{0}}=-\mathbf{0 . 5}$ & $\boldsymbol{V}_{\mathbf{0}}=\mathbf{0}$ & $\boldsymbol{V}_{\mathbf{0}}=\mathbf{0 . 5}$ \\
\hline \multirow{2}{*}{$1 / 3$} & 0.5 & 1.1722 & 0.8264 & 0.5748 & 0.3232 & 0.7232 & 0.4290 & 0.4179 & 0.4068 \\
& 1.0 & 0.9057 & 0.8301 & 0.4425 & 0.0550 & 0.5605 & 0.3432 & 0.3261 & 0.3090 \\
& 1.5 & 0.6961 & 0.7824 & 0.3382 & -0.1059 & 0.4333 & 0.2741 & 0.2545 & 0.2349 \\
$1 / 2$ & 0.5 & 0.9701 & 0.6896 & 0.4680 & 0.2464 & 0.5733 & 0.3408 & 0.3309 & 0.3211 \\
& 1.0 & 0.7493 & 0.7012 & 0.3599 & 0.0186 & 0.4443 & 0.2734 & 0.2582 & 0.2430 \\
& 1.5 & 0.5758 & 0.6657 & 0.2745 & -0.1167 & 0.3434 & 0.2189 & 0.2015 & 0.1841 \\
& 0.5 & 0.4678 & 0.3417 & 0.2088 & 0.0758 & 0.2280 & 0.1369 & 0.1308 & 0.1247 \\
& 1.0 & 0.3611 & 0.3644 & 0.1596 & -0.0450 & 0.1766 & 0.1114 & 0.1020 & 0.9258 \\
& 1.5 & 0.2771 & 0.3553 & 0.1207 & -0.1138 & 0.1365 & 0.0904 & 0.7955 & 0.0687 \\
\hline
\end{tabular}


Table 3: The dimensionless normal stress $\bar{\sigma}_{11}$ in EGP plates $\left(q_{0}=100, \bar{T}_{1}=\bar{T}_{3}=0, \bar{C}_{1}=\bar{C}_{3}=0, \bar{T}_{2}=100, \bar{C}_{2}=2\right)$.

\begin{tabular}{cccccccccc}
\hline \hline \multirow{2}{*}{$\boldsymbol{a} / \boldsymbol{b}$} & $\boldsymbol{k}$ & \multicolumn{9}{c}{$\boldsymbol{a} / \boldsymbol{h}=\mathbf{2}$} & \multicolumn{3}{c}{$\boldsymbol{a} / \boldsymbol{h}=\mathbf{1 0}$} \\
\cline { 3 - 9 } & & $\boldsymbol{F G}$ & $\boldsymbol{V}_{\mathbf{0}}=-\mathbf{0 . 5}$ & $\boldsymbol{V}_{\mathbf{0}}=\mathbf{0}$ & $\boldsymbol{V}_{\mathbf{0}}=\mathbf{0 . 5}$ & $\boldsymbol{F G}$ & $\boldsymbol{V}_{\mathbf{0}}=-\mathbf{0 . 5}$ & $\boldsymbol{V}_{\mathbf{0}}=\mathbf{0}$ & $\boldsymbol{V}_{\mathbf{0}}=\mathbf{0 . 5}$ \\
\hline $1 / 3$ & 0.5 & 1.4383 & 1.4460 & 1.2670 & 1.0881 & 6.3022 & 5.7601 & 5.7241 & 5.6880 \\
& 1.0 & 1.7085 & 1.7859 & 1.4639 & 1.1419 & 7.4271 & 6.6412 & 6.5766 & 6.5121 \\
& 1.5 & 2.0216 & 2.2925 & 1.6861 & 1.0797 & 8.7298 & 7.6770 & 7.5562 & 7.4353 \\
$1 / 2$ & 0.5 & 1.2189 & 1.4306 & 1.1001 & 0.7695 & 5.2003 & 4.9286 & 4.8619 & 4.7951 \\
& 1.0 & 1.4490 & 1.7440 & 1.2723 & 0.8006 & 6.1262 & 5.6803 & 5.5848 & 5.4893 \\
& 1.5 & 1.7156 & 2.2146 & 1.4666 & 0.7185 & 7.2006 & 6.5681 & 6.4170 & 6.2658 \\
1 & 0.5 & 0.6841 & 1.4517 & 0.6695 & -0.1127 & 2.5027 & 2.7786 & 2.6197 & 2.4608 \\
& 1.0 & 0.8169 & 1.6922 & 0.7789 & -0.1344 & 2.9439 & 3.1954 & 3.0072 & 2.8190 \\
& 1.5 & 0.9699 & 2.0600 & 0.9010 & -0.2579 & 3.4602 & 3.6981 & 3.4564 & 3.2147 \\
\hline
\end{tabular}

Table 4: The dimensionless normal stress $\bar{\sigma}_{22}$ in EGP plates $\left(q_{0}=100, \bar{T}_{1}=\bar{T}_{3}=0, \bar{C}_{1}=\bar{C}_{3}=0, \bar{T}_{2}=100, \bar{C}_{2}=2\right)$.

\begin{tabular}{cccccccccc}
\hline \hline \multirow{2}{*}{$\boldsymbol{a} / \boldsymbol{b}$} & $\boldsymbol{k}$ & \multicolumn{9}{c}{$\boldsymbol{a} / \boldsymbol{h}=\mathbf{2}$} & \multicolumn{3}{c}{$\boldsymbol{a} / \boldsymbol{h}=\mathbf{1 0}$} \\
\cline { 3 - 9 } & & FG & $\boldsymbol{V}_{\mathbf{0}}=-\mathbf{0 . 5}$ & $\boldsymbol{V}_{\mathbf{0}}=\mathbf{0}$ & $\boldsymbol{V}_{\mathbf{0}}=\mathbf{0 . 5}$ & $\boldsymbol{F G}$ & $\boldsymbol{V}_{\mathbf{0}}=-\mathbf{0 . 5}$ & $\boldsymbol{V}_{\mathbf{0}}=\mathbf{0}$ & $\boldsymbol{V}_{\mathbf{0}}=\mathbf{0 . 5}$ \\
\hline $1 / 3$ & 0.5 & 0.6986 & 2.2745 & 0.8808 & -0.5129 & 2.6542 & 3.9477 & 3.6658 & 3.3839 \\
& 1.0 & 0.8285 & 2.5440 & 1.0207 & -0.5025 & 3.1040 & 4.5114 & 4.1991 & 3.8868 \\
& 1.5 & 0.9803 & 2.9258 & 1.1809 & -0.5639 & 3.6446 & 5.1901 & 4.8270 & 4.4639 \\
$1 / 2$ & 0.5 & 0.7239 & 2.0841 & 0.8435 & -0.3970 & 2.7683 & 3.7415 & 3.4904 & 3.2392 \\
& 1.0 & 0.8599 & 2.3483 & 0.9782 & -0.3919 & 3.2441 & 4.2819 & 4.0006 & 3.7193 \\
& 1.5 & 1.0181 & 2.7285 & 1.1314 & -0.4656 & 3.8103 & 4.9313 & 4.5986 & 4.2658 \\
1 & 0.5 & 0.6841 & 1.4517 & 0.6695 & -0.1127 & 2.5027 & 2.7786 & 2.6197 & 2.4608 \\
& 1.0 & 0.8169 & 1.6922 & 0.7789 & -0.1344 & 2.9439 & 3.1954 & 3.0072 & 2.8190 \\
& 1.5 & 0.9699 & 2.0600 & 0.9010 & -0.2579 & 3.4602 & 3.6981 & 3.4564 & 3.2147 \\
\hline
\end{tabular}

Table 5: The dimensionless stress $\bar{\sigma}_{12}$ in EGP plates $\left(q_{0}=100, \bar{T}_{1}=\bar{T}_{3}=0, \bar{C}_{1}=\bar{C}_{3}=0, \bar{T}_{2}=100, \bar{C}_{2}=2\right)$.

\begin{tabular}{cccccccccc}
\hline \hline \multirow{2}{*}{$\boldsymbol{a} / \boldsymbol{b}$} & $\boldsymbol{k}$ & \multicolumn{9}{c}{$\boldsymbol{a} / \boldsymbol{h}=\mathbf{2}$} & \multicolumn{3}{c}{$\boldsymbol{a} / \boldsymbol{h}=\mathbf{1 0}$} \\
\cline { 3 - 9 } & & FG & $\boldsymbol{V}_{\mathbf{0}}=-\mathbf{0 . 5}$ & $\boldsymbol{V}_{\mathbf{0}}=\mathbf{0}$ & $\boldsymbol{V}_{\mathbf{0}}=\mathbf{0 . 5}$ & $\boldsymbol{F G}$ & $\boldsymbol{V}_{\mathbf{0}}=-\mathbf{0 . 5}$ & $\boldsymbol{V}_{\mathbf{0}}=\mathbf{0}$ & $\boldsymbol{V}_{\mathbf{0}}=\mathbf{0 . 5}$ \\
\hline \multirow{2}{*}{$1 / 3$} & 0.5 & -0.2758 & 0.3112 & -0.1438 & -0.5989 & 0.7061 & 0.5113 & 0.4250 & 0.3387 \\
& 1.0 & -0.3266 & 0.2861 & -0.1639 & -0.6140 & 0.6502 & 0.4883 & 0.4051 & 0.3219 \\
& 1.5 & -0.3821 & 0.2426 & -0.1839 & -0.6104 & 0.5923 & 0.4593 & 0.3798 & 0.3002 \\
$1 / 2$ & 0.5 & -0.3272 & 0.4368 & -0.1692 & -0.7753 & 0.8362 & 0.6181 & 0.5030 & 0.3880 \\
& 1.0 & -0.3868 & 0.4061 & -0.1921 & -0.7905 & 0.7699 & 0.5904 & 0.4794 & 0.3685 \\
& 1.5 & -0.4502 & 0.3518 & -0.2136 & -0.7790 & 0.7013 & 0.5555 & 0.4494 & 0.3434 \\
& 1.5 & -0.2607 & 0.6234 & -0.1304 & -0.8844 & 0.6504 & 0.5339 & 0.3901 & 0.2462 \\
& 0.5 & -0.3068 & 0.5924 & -0.1462 & -0.8850 & 0.5990 & 0.5107 & 0.3720 & 0.2332 \\
& 1.0 & -0.3524 & 0.5317 & -0.1575 & -0.8469 & 0.5454 & 0.4814 & 0.3487 & 0.2160 \\
\hline
\end{tabular}

Table 6: The dimensionless deflection of and stresses in FGP plates $\left(q_{0}=100, \bar{T}_{1}=\bar{T}_{3}=0, \bar{C}_{1}=\bar{C}_{3}=0, \bar{T}_{2}=100, \bar{C}_{2}=2, V_{0}=-0.5\right)$.

\begin{tabular}{cccccccccc}
\hline \hline \multirow{2}{*}{$\boldsymbol{k}$} & \multicolumn{3}{c}{$\boldsymbol{a} / \boldsymbol{h}=\mathbf{5}$} & \multicolumn{3}{c}{$\boldsymbol{a} / \boldsymbol{h}=\mathbf{1 0}$} & \multicolumn{3}{c}{$\boldsymbol{a} / \boldsymbol{h}=\mathbf{2 0}$} \\
\cline { 2 - 9 } & $\overline{\boldsymbol{w}}$ & $\overline{\boldsymbol{\sigma}}_{\mathbf{1 1}}$ & $\overline{\boldsymbol{\sigma}}_{\mathbf{3 3}}$ & $\overline{\boldsymbol{w}}$ & $\overline{\boldsymbol{\sigma}}_{\mathbf{1 1}}$ & $\overline{\boldsymbol{\sigma}}_{\mathbf{3 3}}$ & $\overline{\boldsymbol{w}}$ & $\overline{\boldsymbol{\sigma}}_{\mathbf{1 1}}$ & $\overline{\boldsymbol{\sigma}}_{\mathbf{3 3}}$ \\
\hline ceramic & 0.18344 & 0.8776 & -3.6692 & 0.16789 & 2.1380 & -1.9652 & 0.16387 & 4.4688 & -1.2452 \\
1 & 0.20830 & 0.9301 & -3.7520 & 0.21143 & 2.4227 & -2.2562 & 0.21234 & 5.1285 & -1.8898 \\
2 & 0.21498 & 1.0374 & -3.8338 & 0.22347 & 2.5620 & -2.3813 & 0.22571 & 5.3700 & -2.1220 \\
3 & 0.22318 & 1.1308 & -3.9006 & 0.23025 & 2.6654 & -2.4664 & 0.23212 & 5.5335 & -2.2664 \\
4 & 0.23103 & 1.2091 & -3.9570 & 0.23547 & 2.7509 & -2.5347 & 0.23658 & 5.6680 & -2.3800 \\
5 & 0.23808 & 1.2748 & -4.0044 & 0.23989 & 2.8245 & -2.5931 & 0.24034 & 5.7880 & -2.4788 \\
6 & 0.24428 & 1.3303 & -4.0440 & 0.24375 & 2.8895 & -2.6420 & 0.24357 & 5.8935 & -2.5648 \\
7 & 0.24967 & 1.3782 & -4.0758 & 0.24713 & 2.9461 & -2.6857 & 0.24639 & 5.9865 & -2.6384 \\
8 & 0.25369 & 1.4187 & -4.0944 & 0.24999 & 2.9942 & -2.7173 & 0.24890 & 6.0685 & -2.7009 \\
9 & 0.22865 & 1.4183 & -3.6682 & 0.24542 & 3.0230 & -2.5193 & 0.24942 & 6.1320 & -2.6432 \\
metal & 0.34951 & 0.8495 & -3.6552 & 0.30302 & 2.1237 & -1.9589 & 0.29119 & 4.4614 & -1.2422 \\
\hline
\end{tabular}




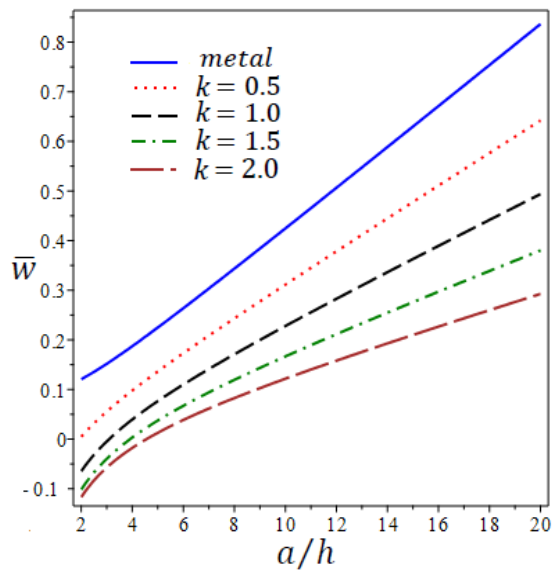

Figure 2: Dimensionless transverse deflection $\bar{w}(0.5,0.5)$ vs the side-to-thickness ratio $a / h$ of the FGP plate $\left(a / b=2, V_{0}=1\right)$.

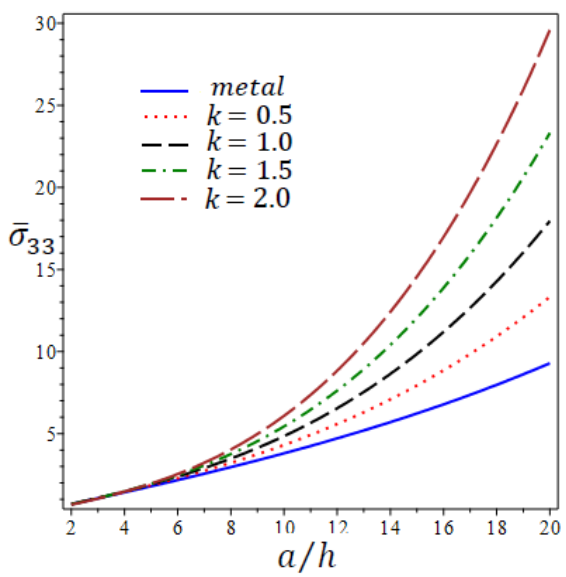

Figure 3: Dimensionless normal stress $\bar{\sigma}_{33}(0.5,0.5,0.5)$ vs the side-to-thickness ratio $a / h$ in the FGP plate $\left(a / b=2, V_{0}=1\right)$.

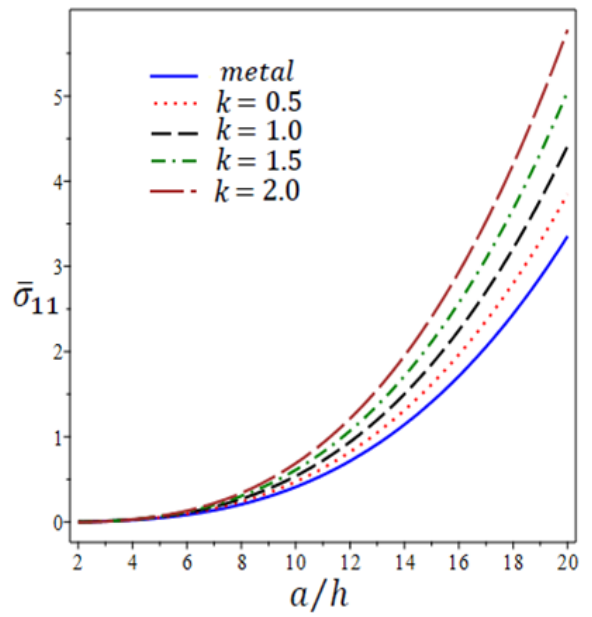

Figure 4: Dimensionless normal stress $\bar{\sigma}_{11}(0.5,0.5,0.5)$ vs the side-to-thickness ratio $a / h$ of FGP $\left(a / b=2, V_{0}=1\right)$.

Figure 2 illustrates the change of normalized transverse deflection $\bar{w}$ with the thickness ratio $a / h$ of the FGP plate when $a / b=2$ and $V_{0}=1$. The dimensionless deflection increases as $a / h$ increases and $k$ decreases. The metallic plate gives the highest deflection. Figures 3 and 4 illustrate the change of normalized normal stresses $\bar{\sigma}_{11}$ and $\bar{\sigma}_{33}$ with the thickness ratio $a / h$ in the FGP plate when $a / b=2, V_{0}=1$. Both stresses increase as $a / h$ and $k$ increase. The metallic plate gives the smallest normal stresses. Figure 5 shows the change of electric displacement $\bar{D}_{3}$ vs the thickness ratio $a / h$ of the FGP rectangular plate. The electric displacement increases as $a / h$ increases for homogeneous metallic plate while the electric displacement FGP rectangular plates decreases as $a / h$ increases. Also, $\bar{D}_{3}$ decreases as $k$ increases. 


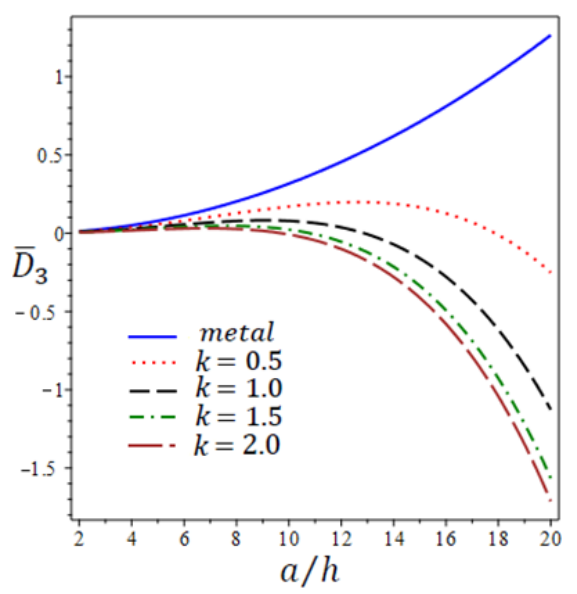

Figure 5: Dimensionless electric displacement $\bar{D}_{3}(0.5,0.5,0)$ vs the side-to-thickness ratio $a / h$ of FGP plate $\left(a / b=2, V_{0}=1\right)$.

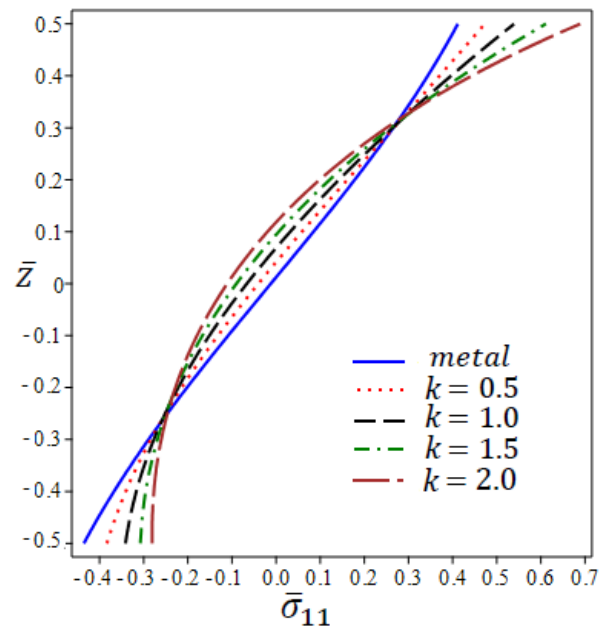

Figure 6: Dimensionless normal stress $\bar{\sigma}_{11}(0.5,0.5, \bar{z})$ through the thickness in the FGP plate $\left(a / b=2, V_{0}=1, a / h=10\right)$.

Figures 6 and 7 illustrate the through the thickness distributions of the longitudinal and transverse normal stresses $\bar{\sigma}_{11}$ and $\bar{\sigma}_{33}$ in the FGP plate. The longitudinal normal stress $\bar{\sigma}_{11}$ is tensile at the top surface and compressive at the bottom surface of the FGP plate. The longitudinal normal stress $\bar{\sigma}_{11}$ increases as $k$ decreases in the region $-0.25 \leq \bar{Z} \leq$ 0.3 and vice versa outside this region. However, the transverse normal stress $\bar{\sigma}_{33}$ is always tensile through the thickness of the FGP plate. Also, the transverse normal stress $\bar{\sigma}_{33}$ increases as $k$ decreases in the region $-0.22 \leq \bar{z} \leq 0.26$ and vice versa outside this region.

Figure 8 shows the change of normalized transverse deflection $\bar{w}$ through the thickness of the FGP plate. The dimensionless deflection $\bar{w}$ may be independent of the thickness of the FGP plate. The metallic plate gives the highest deflection. Otherwise, the deflection decreases as $k$ increases. Figure 9 illustrates the through the thickness distributions of the tangential shear stress $\bar{\sigma}_{12}$ of the FGP plate. The tangential shear stress $\bar{\sigma}_{12}$ is tensile at the bottom surface and compressive at the top surface of the FGP plate. The tangential shear stress $\bar{\sigma}_{12}$ increases as $k$ increases in the region $-0.28 \leq \bar{Z} \leq 0.34$ and vice versa outside this region. 


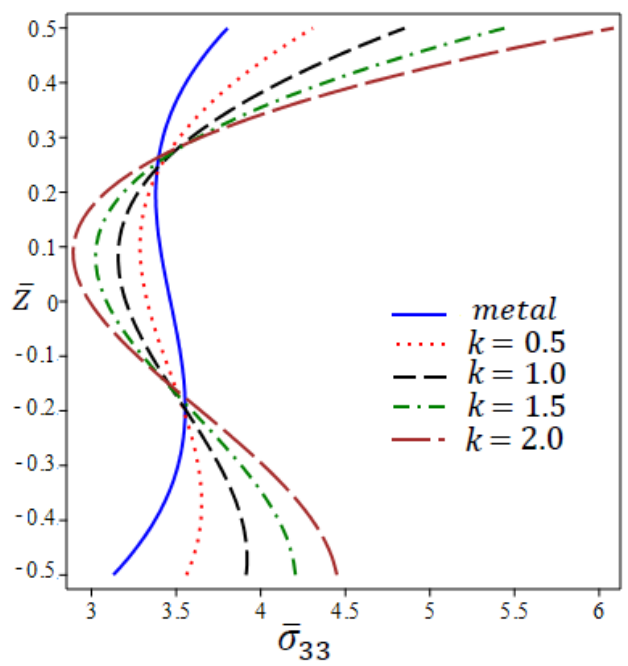

Figure 7: Dimensionless normal stress $\bar{\sigma}_{33}(0.5,0.5, \bar{z})$ through the thickness in the FGP plate $\left(a / b=2, V_{0}=1, a / h=10\right)$.

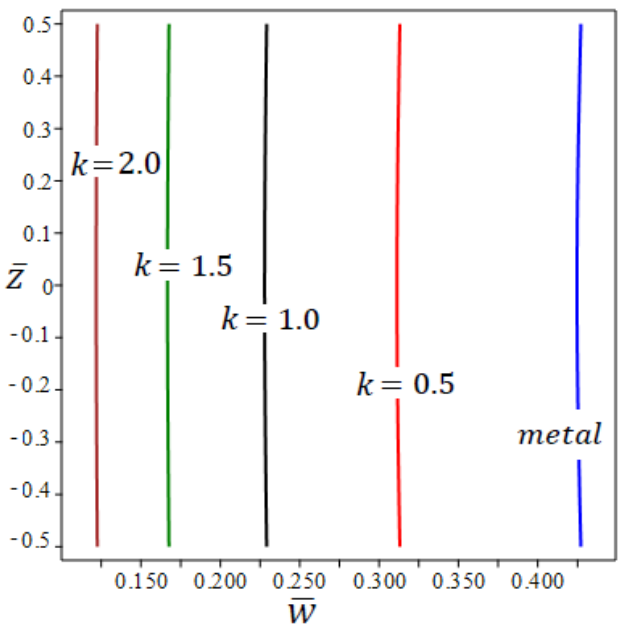

Figure 8: Dimensionless transverse deflection $\bar{w}(0.5,0.5, \bar{z})$ through the thickness of the FGP plate $\left(a / b=2, V_{0}=1, a / h=10\right)$

In what follows, we will discuss the effect of the transverse hygrothermal parameters $\bar{T}_{3}, \bar{C}_{3}$ and the external electric voltage $V_{0}$ on the bending response of FGP plates. Figures 10-17 are studied based on the following five cases:

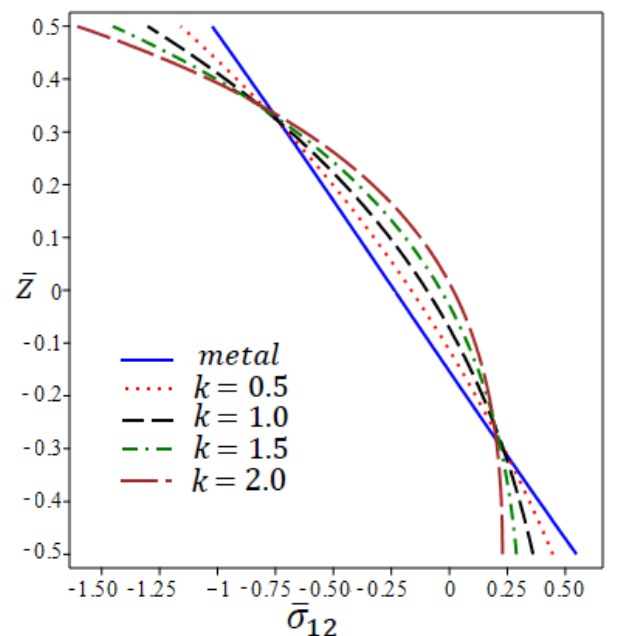

Figure 9: Dimensionless shear stress $\bar{\sigma}_{12}(0,0.5, \bar{z})$ through the thickness in the FGP plate $\left(a / b=2, V_{0}=1, a / h=10\right)$. 


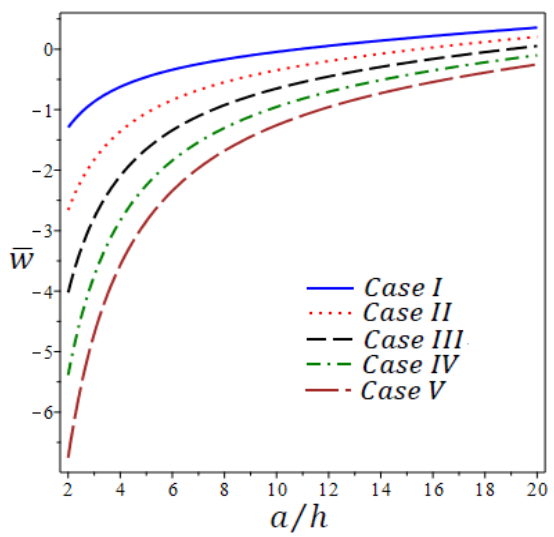

Figure 10: Dimensionless transverse deflection $\bar{w}(0.5,0.5,0)$ vs the side-to-thickness ratio $a / h$ of the FGP plate $(a / b=2, k=1)$.

case I: $\bar{T}_{3}=-100, \bar{C}_{3}=2, V_{0}=10$,

case II: $\bar{T}_{3}=-50, \bar{C}_{3}=10, V_{0}=20$,

case III: $\bar{T}_{3}=50, \bar{C}_{3}=50, V_{0}=30$

case IV: $\bar{T}_{3}=100, \bar{C}_{3}=100, V_{0}=40$,

case $V: \bar{T}_{3}=150, \bar{C}_{3}=150, V_{0}=50$.

Figures 10-13 establish the change of $\bar{w}, \bar{\phi}, \bar{\sigma}_{11}$ and $\bar{D}_{3}$, respectively, with the thickness ratio $a / h$ for different values of $\bar{T}_{3}, \bar{C}_{3}$ and $V_{0}$. The dimensionless deflection $\bar{w}$, electric potential $\bar{\phi}$ and longitudinal normal stress $\bar{\sigma}_{11}$ are increasing with the increase in $\bar{T}_{3}, \bar{C}_{3}$ and $V_{0}$ while the dimensionless electric displacement $\bar{D}_{3}$ is decreasing. The dimensionless deflection $\bar{w}$ and electric displacement $\bar{D}_{3}$ are directly increasing with the increase in the side-to-thickness ratio $a / h$. However, the electric potential $\bar{\phi}$ and longitudinal normal stress $\bar{\sigma}_{11}$ are no longer decreasing as $a / h$ and they are increasing again to get their maximum values for higher values of $a / h$.

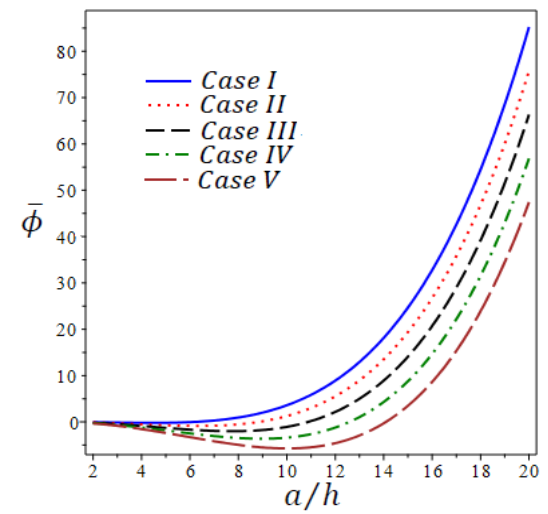

Figure 11: Dimensionless electric potential $\bar{\phi}(0.5,0.5,0)$ vs the side-to-thickness ratio $a / h$ of the FGP plate $(a / b=2, k=1)$.

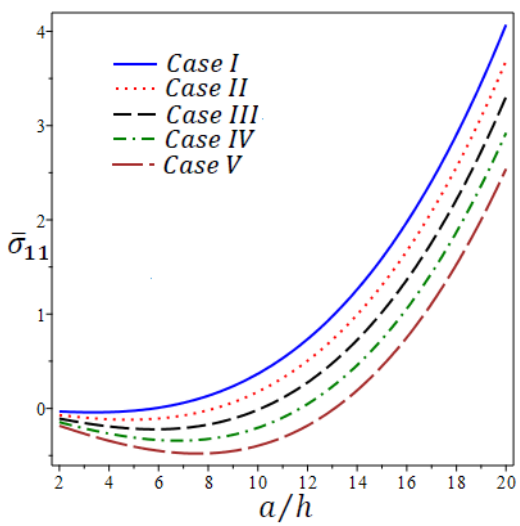

Figure 12: Dimensionless normal stress $\bar{\sigma}_{11}(0.5,0.5,0.5)$ vs the side-to-thickness ratio $a / h$ of the FGP plate $(a / b=2, k=1)$ 
Figure 14 illustrates that the electric potential $\bar{\phi}$ is tensile in the upper-half plane and compressive in the lower-half plane of the FGP plate. The dimensionless electric potential $\bar{\phi}$ is increasing with the increase in $\bar{T}_{3}, \bar{C}_{3}$ and $V_{0}$ in the upperhalf plane and is decreasing in the lower-half plane of the FGP plate. In Figure 15, the electric displacement $\bar{D}_{3}$ increases as $\bar{T}_{3}, \bar{C}_{3}$ and $V_{0}$ increases. For case I, $\bar{D}_{3}$ may be independent of the thickness of the FGP plate. However, for other cases $\bar{D}_{3}$ gets its maximum value at the bottom surface of the FGP plate and its minimum value at the top one.

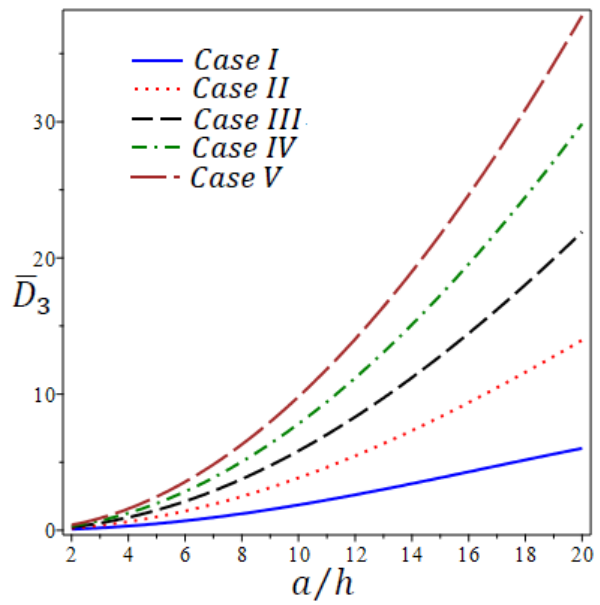

Figure 13: Dimensionless of electric displacement $\bar{D}_{3}(0.5,0.5,0)$ vs the side-to-thickness ratio $a / h$ of the FGP plate $(a / b=2, k=1)$.

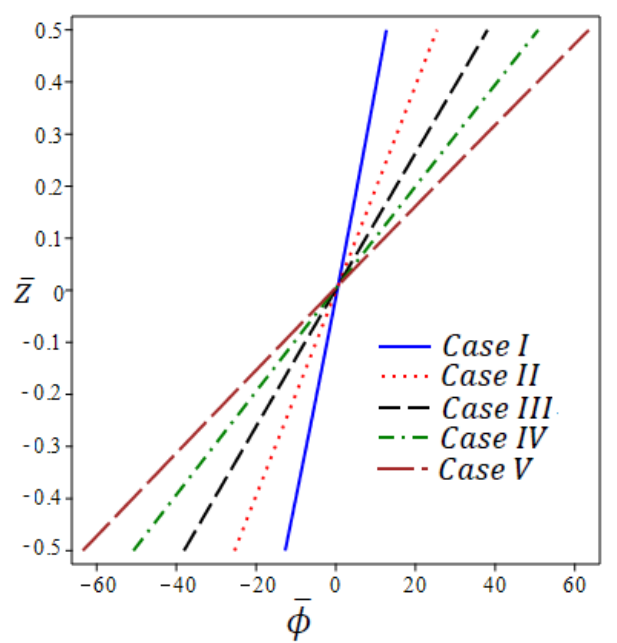

Figure 14: Dimensionless electric potential $\bar{\phi}(0.5,0.5, \bar{z})$ through the thickness of the FGP plate $(a / b=2, a / h=10, k=1)$.

Figure 16 shows that the longitudinal normal stress $\bar{\sigma}_{11}$ increases as $\bar{T}_{3}, \bar{C}_{3}$ and $V_{0}$ decrease. The longitudinal normal stress $\bar{\sigma}_{11}$ is tensile near the top surface for cases I and II and compressive for other cases through-the-thickness the FGP plate. Figure 17 shows that the transverse normal stress $\bar{\sigma}_{33}$ is tensile through-the-thickness of FGP plate and it increases as $\bar{T}_{3}, \bar{C}_{3}$ and $V_{0}$ increase. It is noticed that $\bar{\sigma}_{33}$ has a little dependency on the thickness of the FGP plate.

\section{CONCLUSIONS}

This paper presents analytical solutions for bending of FGP rectangular plates subjected to moisture and thermal loads. The equilibrium equations based on the principle of virtual work, and the analytical closed-form solutions of simply-supported FGP plates are obtained by using Navier's method. The solutions are derived by using a quasi-3D sinusoidal shear and normal deformations theory. The number of primary variables in the present theory is six. 


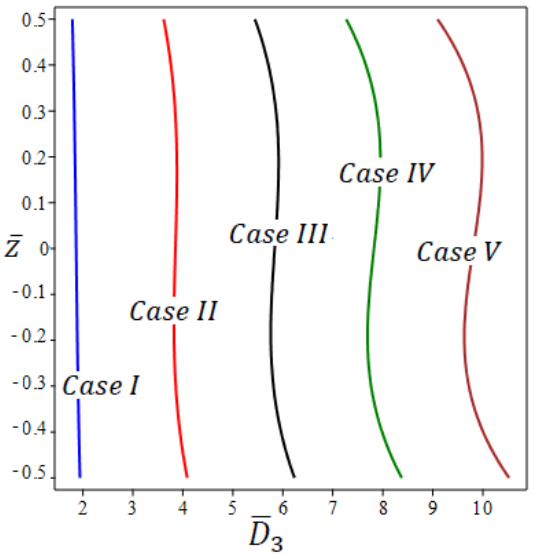

Figure 15: Dimensionless electric displacement $\bar{D}_{3}(0.5,0.5, \bar{z})$ through the thickness of the FGP plate $(a / b=2, a / h=10, k=1)$.

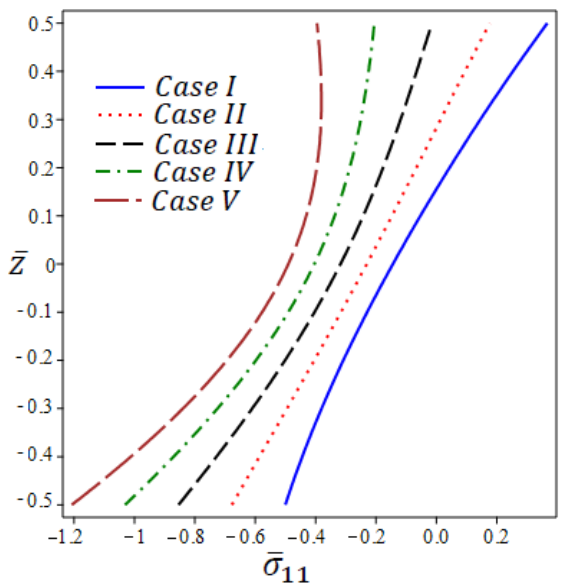

Figure 16: Dimensionless normal stress $\bar{\sigma}_{11}(0.5,0.5, \bar{z})$ through the thickness in the FGP plate $(a / b=2, a / h=10, k=1)$.

The theory satisfies the boundary conditions on the surfaces of the FGP plate without employing shear correction factors. The present FGP plate is subjected to mechanical, thermal, moisture and electric voltage loadings in its top surface. Non-dimensional displacements and stresses are computed for FGP plates with mixture materials. The responses of stress and displacement of the FGP rectangular plate are analyzed under hygro-thermo-mechanical sinusoidal loadings. Material properties are supposed to be dependent on the moisture and temperature effects, which constantly vary through the thickness of the FGP plate. The dependency of the deflection, stresses, electric potential and electric displacement of the FGP plate on different parameters is discussed. It is concluded that the gradients in material properties and the inclusion of other parameters have important roles to play in locating the response of the FGP plate.

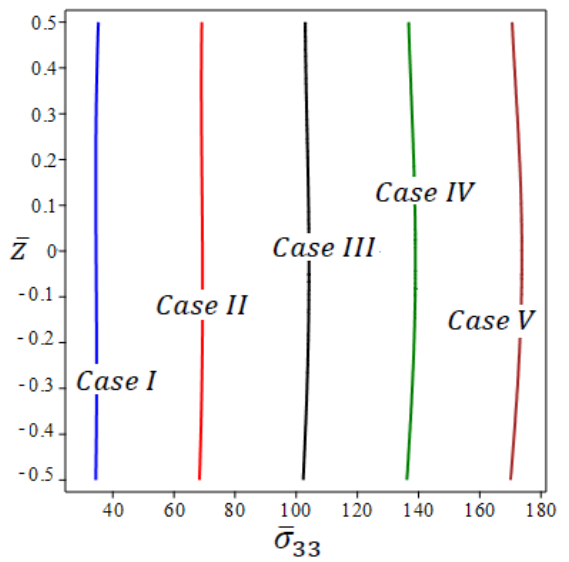

Figure 17: Dimensionless normal stress $\bar{\sigma}_{33}(0.5,0.5, \bar{z})$ through the thickness of the FGP plate $(a / b=2, a / h=10, k=1)$ 
Author's Contributions: Conceptualization, AM Zenkour; Methodology, AM Zenkour; Investigation, AM Zenkour and ZS Hafed; Writing - original draft, ZS Hafed; Writing - review \& editing, AM Zenkour; Funding acquisition, ZS Hafed; Resources, AM Zenkour and ZS Hafed; Supervision, AM Zenkour.

Editor: Pablo Andrés Muñoz Rojas.

\section{References}

Al Khateeb, S.A., Zenkour, A.M., (2014). A refined four-unknown plate theory for advanced plates resting on elastic foundations in hygrothermal environment. Composite Structures 111: 240-248.

Altenbach, H., Eremeyev, V.A., (2008a). Direct approach-based analysis of plates composed of functionally graded materials. Archive of Applied Mechanics 78(10): 775-794.

Altenbach, H., Eremeyev, V.A., (2008b). Analysis of the viscoelastic behavior of plates made of functionally graded materials. Zeitschrift für Angewandte Mathematik und Mechanik (ZAMM) 88(5): 332-341.

Bellifa, H., Benrahou, K.H., Hadji, L., Houari, M.S.A., Tounsi, A., (2016). Bending and free vibration analysis of functionally graded plates using a simple shear deformation theory and the concept the neutral surface position. Journal of the Brazilian Society of Mechanical Sciences and Engineering 38:265-275.

Beni, Y.T., (2016). Size-dependent electromechanical bending, buckling, and free vibration analysis of functionally graded piezoelectric nanobeams. Journal of Intelligent Material Systems and Structures 27: 2199-2215.

Benyoucef, S., Mechab, I., Tounsi, A., Fekrar, A., Atmane, H.A. and Adda Bedia, E.A., (2010). Bending of thick functionally graded plates resting on Winkler-Pasternak elastic foundations. Mechanics of Composite Materials 46: 425-434.

Bîrsan, M., Altenbach, H., Sadowski, T., Eremeyev, V.A., Pietras, D., (2012). Deformation analysis of functionally graded beams by the direct approach. Composites Part B: Engineering 43(3): 1315-1328.

Bouderba, B., Houari, M.S.A., Tounsi, A., (2013). Thermomechanical bending response of FGM thick plates resting on WinklerPasternak elastic foundations. Steel and Composite Structures 14: 85-104.

Bousahla, A.A., Houari, M.S.A., Tounsi, A., Adda Bedia, E.A., (2014). A Novel higher order shear and normal deformation theory based on neutral surface position for bending analysis of advanced composite plates. International Journal of Computational Methods 11: 1350082.

Cheng, Z.Q., Batra, R.C., (2000a). Three-dimensional thermoelastic deformations of a functionally graded elliptic plate. Composites 31: 97-106.

Cheng, Z.Q., Batra, R.C., (2000b). Deflection relationships between the homogeneous Kirchhoff plate theory and different functionally graded plate theories. Archives of Mechanics 52: 143-158.

Cho, M., Oh, J., (2004). A finite element based on cubic zig-zag plate theory for the prediction of thermo-electric-mechanical behaviors. International Journal of Solids and Structures 41: 1357-1375.

Huang, G.Y., Yu, S.W., (2006). Effect of surface piezoelectricity on the electromechanical behavior of a piezoelectric ring. Phys. Physica Status Solidi B 243: 22-24.

Jafari, A.A., Jandaghian, A.A., Rahmani, O., (2014). Transient bending analysis of a functionally graded circular plate with integrated surface piezoelectric layers. International Journal of Mechanical and Materials Engineering 9: 1-14.

Jandaghian, A.A., Jafari, A.A., Rahmani, O., (2013). Exact solution for Transient bending of a circular plate integrated with piezoelectric layers. Applied Mathematical Modelling 37: 7154-7163.

Jandaghian, A.A., Jafari, A.A., Rahmani, O., (2014). Vibrational response of functionally graded circular plate integrated with piezoelectric layers: an exact solution. Engineering Solid Mechanics 2: 119-130.

Jha, D.K., Kant, T., Singh, R.K., (2013). A critical review of recent research on functionally graded plates. Composite Structures 96: 833-849.

Ke, L.L., Liu, C., Wang, Y.S., (2015). Free vibration of nonlocal piezoelectric nanoplates under various boundary conditions. Physica E: Low-dimensional Systems and Nanostructures 66: 93-106. 
Lee, H.J., Saravanos, D.A., (1997). Generalized finite element formulation for smart multilayered thermal piezoelectric composite plates. International Journal of Solids and Structures. 34: 3355-3371.

Li, Y.S., Feng, W.J., Cai, Z.Y., (2014). Bending and free vibration of functionally graded piezoelectric beam based on modified strain gradient theory. Composite Structures 115: 41-50.

Liu, C., Ke, L.L., Wang, Y.S., Yang, J., Kitipornchai, S., (2013). Thermo-electro-mechanical vibration of piezoelectric nanoplates based on the nonlocal theory. Composite Structures 106: 167-174.

Loy, C.T., Lam, K.Y., Reddy, J.N., (1999). Vibration of functionally graded cylindrical shells. International Journal of Mechanical Sciences 41: 309-324.

Lü, C.F., Lim, C.W., Chen, W.Q., (2009). Semi-analytical analysis for multi-directional functionally graded plates: 3-D elasticity solutions. International Journal for Numerical Methods in Engineering 79: 25-44.

Mechab, I., Atmane, H.A., Tounsi, A., Belhadj, H.A., Adda Bedia, E.A., (2010). A two variable refined plate theory for the bending analysis of functionally graded plates. Acta Mechanica Sinica 26: 941-949.

Mindlin, R.D., (1957). Influence of rotatory inertia and shear on flexural motions of isotropic, elastic plates. Journal of Applied Mechanics 18: 31-38.

Noda, N., (1999). Thermal stresses in functionally graded materials. Journal of Thermal Stresses 22: 477-512.

Ootao, Y., Tanigawa, Y., (2000). Three-dimensional transient piezothermoelasticity in functionally graded rectangular plate bonded to a piezoelectric plate. International Journal of Solids and Structures 37: 4377-401.

Qian, L.F., Batra, R.C., (2004). Transient thermoelastic deformations of a thick functionally graded plate. Journal of Thermal Stresses 27: 705-740.

Reddy, J.N., (1984). A simple higher-order theory for laminated composite plates. Journal of Applied Mechanics 51: 745-752.

Reddy, J.N., (2000). Analysis of functionally graded plates. International Journal for Numerical Methods in Engineering 47: 663-684.

Reddy, J.N., Chin, C.D., (1998). Thermomechanical analysis of functionally graded cylinders and plates. Journal of Thermal Stresses 21: 593-626.

Reissner, E., (1945). The effect of transverse shear deformation on the bending of elastic plates. Journal of Applied Mechanics 12: 69-72.

Tahani, M., Mirzababaee, S.M., (2009). Non-linear analysis of functionally graded plates in cylindrical bending under thermomechanical loadings based on a layer-wise theory. Eur. Journal of Mechanics A/Solids 28: 248-256.

Thai, H.T., Vo, T.P., (2013). A new sinusoidal shear deformation theory for bending, buckling, and vibration of functionally graded plates. Applied Mathematical Modelling 37: 3269-81.

Tiersten, H.F., (1969). Linear Piezoelectric Plate Vibrations. New York, NY: Plenum.

Tounsi, A., Houari, M.S.A., Benyoucef, S., (2013). A refined trigonometric shear deformation theory for thermoelastic bending of functionally graded sandwich plates. Aerospace Science and Technology 24: 209-220.

Touratier, M., (1991). An efficient standard plate theory. International Journal of Engineering Science 29: 901-916.

Vel, S.S., Batra, R.C., (2002). Exact solution for thermoelastic deformations of functionally graded thick rectangular plates. AIAA Journal 40: 1421-1433.

Yang, J., Shen, H.S., (2001). Dynamic response of initially stressed functionally graded rectangular thin plates. Composite Structures 54: 497-508.

Ying, J., Lü, C.F., Lim, C.W., (2009). 3D thermoelasticity solutions for functionally graded thick plates. Journal of Zhejiang University-SCIENCE A 10: 327-336.

Zenkour, A.M., (2006). Generalized shear deformation theory for bending analysis of functionally graded plates. Applied Mathematical Modelling 30: 67-84.

Zenkour, A.M., (2007). Benchmark trigonometric and 3-D elasticity solutions for an exponentially graded thick rectangular plate. Archive of Applied Mechanics 77: 197-214. 
Zenkour, A.M., (2014a). Hygrothermoelastic responses of inhomogeneous piezoelectric and exponentially graded cylinders. International Journal of Pressure Vessels and Piping 119: 8-18.

Zenkour, A.M., (2014b). Exact solution of thermal stress problem of an inhomogeneous hygrothermal piezoelectric hollow cylinder. Applied Mathematical Modelling 38: 6133-6143.

Zenkour, A.M., Hafed, Z.S., (2019). Bending analysis of functionally graded piezoelectric plates via quasi-3D trigonometric theory. Mechanics of Advanced Materials and Structures, In Press, DOI: 10.1080/15376494.2018.1516325.

Zenkour, A.M, Sobhy, M., (2015). Simplified shear and normal deformations nonlocal theory for bending of nanobeams in thermal environment. Physica E 70: 121-128.

Zhang, D.G., Zhou, Y.H., (2008). A theoretical analysis of FGM thin plates based on physical neutral surface. Computational Materials Science 44: 716-720.

Zidi, M., Tounsi, A., Houari, M.S.A., Bég, O.A., (2014). Bending analysis of FGM plates under hygro-thermo-mechanical loading using a four variable refined plate theory. Aerospace Science and Technology 34: 24-34. 